\title{
Hf isotopes in zircon from the western Superior province, Canada: Implications for Archean crustal development and evolution of the depleted mantle reservoir
}

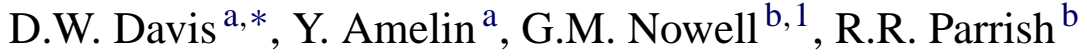 \\ a Department of Geology, University of Toronto, 22 Russell Street, Toronto, Ont., Canada M5S 3B1 \\ b NERC Isotope Geoscience Laboratory, Kingsley Dunham Centre, Keyworth, Nottingham NG12 5GG, UK
}

Received 24 August 2004; received in revised form 15 July 2005; accepted 25 July 2005

\begin{abstract}
$\mathrm{U}-\mathrm{Pb}$ and $\mathrm{Hf}$ isotopic measurements on zircons from the western Superior province confirm that the area contains at least three distinct terrane types. Juvenile terranes that formed mostly within the time span 2.75-2.68 Ga occupy much of the western Wabigoon subprovince as well as granite-greenstone belts to the south. Juvenile $3.0 \mathrm{Ga}$ terranes that were reworked over the time span 2.7-3.0 Ga occupy the south-central part of the Wabigoon subprovince and the North Caribou block in the Sachigo subprovince. Rocks with mantle extraction ages as old as $3.5 \mathrm{Ga}$ and zircon $\mathrm{U}-\mathrm{Pb}$ ages extending to $3.3 \mathrm{Ga}$ characterize a third type of terrane represented by the Winnipeg River subprovince. This terrane was strongly reworked during the late Archean.

Arc-related magmatism was ongoing at 2.71-2.75 Ga in the different terranes, which probably accreted over the time span 2.71-2.68 Ga. Enriched Hf and high O isotopic signatures in late sanukitoid-suite plutons appear to be correlated, which suggests that assimilation of Mesoarchean crust was an important factor in their magmatic evolution.

Enriched Hf isotopic signatures in detrital and igneous zircon from parts of the north-central Wabigoon subprovince support previous suggestions that the Winnipeg River terrane extends eastward beyond the Winnipeg River subprovince. The Winnipeg River subprovince was probably being uplifted and eroded into the Quetico sedimentary basin shortly after $2700 \mathrm{Ma}$, as shown by detrital zircons with enriched Hf isotopic signatures and Meso- to Paleoarchean ages. The pattern of ages and isotopic signatures from the North Caribou block and the south-central Wabigoon subprovince are similar, suggesting that these terranes are correlative. If so, the south-central Wabigoon terrane may have been tectonically transported from the north.

$\mathrm{Hf}$ isotopic compositions of zircon from juvenile Archean sources are remarkably consistent and define an average $\varepsilon_{\mathrm{Hf}}$ value of $+3.5 \pm 0.2$ for samples with an average age of $2724 \mathrm{Ma}$ and a best estimate of $+2.7 \pm 0.4$ at 3000 Ma. Thus, the Neaoarchean depleted mantle reservoir beneath the Superior province appears to have been isotopically well mixed. $\varepsilon_{\mathrm{Hf}}$ values were calculated using a value of $1.865 \times 10^{-5} \mathrm{Ma}^{-1}$ [Scherer, E., Munker, C., Mezger, K., 2001. Calibration of the Lutetium-Hafnium clock. Science 293, 683-687] for the ${ }^{176} \mathrm{Lu}$ decay constant, which is thus far the best reproduced estimate and the one most consistent with depleted mantle evolution results based on $\mathrm{Nd}$ isotopes and $\mathrm{Nb} / \mathrm{Th}$ ratios. A linear $\mathrm{Hf}$ mantle growth curve defined by these

\footnotetext{
* Corresponding author. Tel.: +1 416946 0365; fax: +1 4169783938.

E-mail address: dond@geology.utoronto.ca (D.W. Davis).

1 Present address: Arthur Holmes Isotope Geology Laboratory, Department of Geological Sciences, University of Durham, South Road, Durham DH1 3LE, UK.
} 
values and recent MORB intersects the chondritic Hf growth curve during the early Archean (3.4-4.0 Ga). This could indicate that the earliest formation of significant amounts of enriched crust coincides with ages of the oldest preserved rocks, but such a conclusion is contradicted by evidence from ${ }^{142} \mathrm{Nd}$ and ${ }^{143} \mathrm{Nd}$ in early Archean rocks for significant mantle depletion during the Hadean eon $(>4.0 \mathrm{Ga})$. Both lines of evidence might be reconciled if Hadean enriched crust were largely remixed with its depleted mantle source near the beginning of the Archean, leaving only fragmentary evidence of its existence in the oldest rocks. (C) 2005 Elsevier B.V. All rights reserved.

Keywords: Superior province; $\mathrm{Hf}$ isotopes Archean; Zircon; U-Pb geochronology

\section{Introduction}

$\mathrm{U}-\mathrm{Pb}$ dating of zircon in the Superior province has revealed a near continuous record of magmatism within the time range $2650-3000 \mathrm{Ma}$ but the vast majority of rocks formed within the last $100 \mathrm{~m}$.y. of this period. Precise dating of these rocks has contributed important information toward understanding Neoarchean tectonic processes, in particular showing that the Superior province consists of terranes with distinct histories and degrees of crustal reworking. Age data on zircons from igneous and sedimentary rocks (Davis, 1998) together with field and geophysical observations (White et al., 2003) in the context of the western Superior Lithoprobe transect, support earlier proposals that the Superior province was assembled from successive collisions with such terranes (Langford and Morin, 1976; Card, 1990).

Nd isotopic work by Henry et al. $(1998,2000)$ and Tomlinson et al. (2003) has greatly helped in recognizing and delineating the extent of different terranes in the Superior province. Variations in the ${ }^{176} \mathrm{Hf}$ isotope from decay of ${ }^{176} \mathrm{Lu}$ can distinguish juvenile mantle from older crustal sources in a way similar to $\mathrm{Nd}$ isotopes (Patchett, 1983). It has been shown that Hf isotopic variations are closely coupled to $\mathrm{Nd}$ isotopes in the crust and mantle (Blichert-Toft and Albarède, 1997; Vervoort and Blichert-Toft, 1999) but measurements of $\mathrm{Hf}$ isotopes in zircon have some advantage over $\mathrm{Nd}$ isotope measurements on whole rocks. Zircon contains Hf as a major (1-2\%) and highly immobile component, so there is less possibility of disturbance and minimal age correction is required to arrive at the primary $\mathrm{Hf}$ isotopic ratio. $\mathrm{U}-\mathrm{Pb}$ dating also provides a precise age of crystallization on the same sample used for Hf measurement. The development of multi-collector mass spectrometers with inductively couple plasma sources (MC-ICPMS) has greatly facilitated rapid and precise analysis of Hf isotopes in small zircon samples including analysis of single detrital grains that can potentially reveal the magmatic pre-history of sedimentary source terrains.

Hf isotopes contained within collections of dated zircon provide a rich potential source of data. The Jack Satterly Geochronology Laboratory has archived zircon from thousands of previously dated rocks. Using MC-ICPMS, Hf isotopes were measured on dated zircon from 62 igneous rock units plus 25 detrital zircon grains from Archean metasedimentary rocks in the western Superior province. Most samples come from northwest Ontario. The mass spectrometer analyses were done over a four-day period and expand the previously available Hf database in the region by a factor of four. The objective of this study was to acquire data over as wide an area as possible to test whether Hf isotopic compositions can be used to identify distinct tectonic terranes. Samples were selected from seven different areas of the western Superior province that may represent terranes with distinctly different histories. They are from a variety of lithologies, including volcanic rocks, syn-volcanic plutons, gneisses, late plutons, and detrital zircons. The zircons cover an age range of greater than $600 \mathrm{~m}$.y. The data present a broad picture of terrane histories in the western Superior province and in some cases allow sources of detrital zircon to be isotopically distinguished. Pending acceptance of a well established value for the ${ }^{176} \mathrm{Lu}$ decay constant, the data also help constrain evolution of late Archean depleted mantle beneath the region, which has a bearing on global development of the early continental crust.

\section{Background}

The Superior province has been divided into subprovinces based on contrasts in principal lithologies 


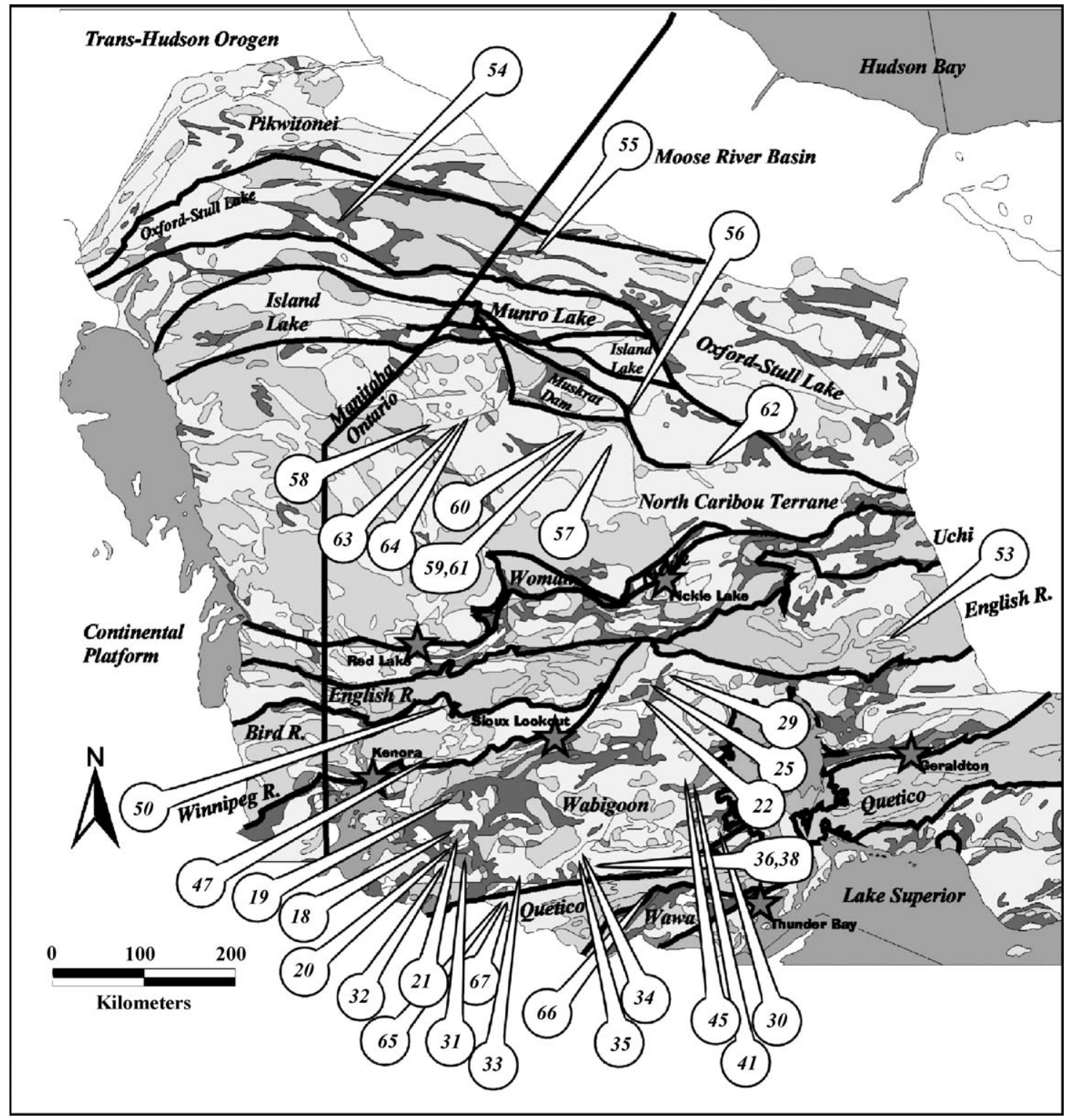

Fig. 1. Geological map of the western Superior province showing sample locations. Subprovince divisions are after Card and Ciesielski (1986).

and metamorphic grade (Fig. 1 based on Card and Ciesielski, 1986). The northern part of the study area contains the granite-greenstone Sachigo and Uchi subprovinces, the metasedimentary English River subprovince, and the metaplutonic Winnipeg River subprovince. The latter two areas are generally metamorphosed to mid-amphibolite or granulite facies.
Further south is the granite-greenstone Wabigoon subprovince, followed by the metasedimentary Quetico subprovince and finally the granite-greenstone Shebandowan belt. The Shebandowan belt is the western extension of the Wawa subprovince, which is probably correlative with the Abitibi subprovince further east. 
Smith et al. (1987) followed by Stevensen and Patchett (1990) and Corfu and Noble (1992) presented the earliest studies of Hf isotopic ratios in the Superior province. These confirmed the juvenile character of igneous rocks in the southern Abitibi and Wawa subprovinces, already suspected from $\mathrm{U}-\mathrm{Pb}$ dating. However, $\mathrm{Hf}$ isotopes in bulk detrital zircon fractions from a number of Archean sandstones indicated that rocks as old as about $3.0 \mathrm{Ga}$ were probably present in the provenance (Stevensen and Patchett, 1990). Later Hf measurements by Corfu and Stott $(1993 a, 1996)$ in the Uchi subprovince showed a distribution of $\varepsilon_{\mathrm{Hf}}$ values that range between depleted mantle and $3.0 \mathrm{Ga}$ crustal growth curves. Based on $\mathrm{U}-\mathrm{Pb}$ geochronology and field investigations, the Uchi subprovince was previously suggested to have developed over a 300 m.y. period in an Andean-type tectonic setting along the margin of a $3.0 \mathrm{Ga}$ old terrane to the north, the North Caribou terrane (Stott and Corfu, 1991; Thurston et al., 1991). The early Hf isotopic data support this model and provide evidence that the North Caribou terrane contains no components significantly older than $3.0 \mathrm{Ga}$.

Other studies based on $\mathrm{U}-\mathrm{Pb}$ age and $\mathrm{Nd}$ isotopic measurements have shown that the western Superior province contains a number of terranes with different histories. The restricted time span for igneous activity in the greenstone-dominated western part of the Wabigoon subprovince and the absence of older zircon inheritance led Davis et al. (1988) to suggest that this was a juvenile oceanic arc that began to form at $2.77 \mathrm{Ga}$ and accreted at $2.70-2.71 \mathrm{Ga}$. A similar history has been found for the Shebandowan greenstone belt (Corfu and Stott, 1998, 1993b), which is co-extensive with the Wawa and Abitibi subprovinces to the east. In contrast, the Sachigo and Winnipeg River subprovinces contain abundant Mesoarchean rocks. The Winnipeg River subprovince contains some of the oldest rocks yet dated in the region, with gneisses as old as $3310 \mathrm{Ma}$ (Melnyk et al., submitted) and Nd model ages extending back to $3.4 \mathrm{Ga}$ (Henry et al., 2000; Dickin et al., 1990). The boundaries of these different terranes are not always coincident with subprovince boundaries. The reworked 3.0 Ga North Caribou terrane comprises parts of the Sachigo subprovince and probably extends into the northern margin of the Uchi subprovince. The south-central part of the Wabigoon subprovince is also suggested to have formed from reworking of a juvenile 3.0 Ga terrain (Davis and Jackson, 1988; Tomlinson et al., 2003) while Nd isotopes from the north-central part show evidence for older Mesoarchean sources that may be correlative with rocks in the Winnipeg River subprovince (Tomlinson et al., 2003).

Mesoarchean terranes in the Superior province characteristically contain locally developed units of quartzrich arenite. Dated detrital zircons from these units are all older than $2.8 \mathrm{Ga}$, pre-dating the most intense period of arc-related magmatism, which began at about $2750 \mathrm{Ma}$. Their detrital zircos generally reflect the ages of nearby Mesoarchean igneous rocks. These units are suggested to be indicative of an early period of continental stability and development of platform sequences (Thurston and Chivers, 1990).

Some of the most important evidence for tectonic processes comes from $\mathrm{U}-\mathrm{Pb}$ ages of detrital zircons in sandstones and conglomerates from the greenstone belts and from metaturbidites that make up the supracrustal component of the Quetico and English River subprovinces and the Pontiac subprovince in the southeastern Superior province. Their detrital zircons constrain sediment deposition to the period 2.712.68 Ga (Davis, 1998, 2002; Davis et al., 1990). Depositional ages appear to become younger from north to south across the southern Superior province. They generally correspond to the early part of regional deformation in any given area and locally post-date calcalkaline and tholeiitic volcanism by several million years (Davis, 1998, 2002). Based partly on this evidence, it has been proposed that the Superior province was built by accretion of arcs and continental fragments from the south over this time period (Percival and Williams, 1989). This process was coeval with emplacement of syn and late tectonic 'sanukitoid suite' plutons derived from melting of previously metasomatized mantle (Shirey and Hanson, 1984; Stern et al., 1990).

\section{Analytical methods}

Many of the samples were dated in previous studies by $\mathrm{U}-\mathrm{Pb}$ analysis of zircon at the Royal Ontario Museum. In some cases washes from the $\mathrm{U}-\mathrm{Pb}$ columns, which contain $\mathrm{Zr}$ and $\mathrm{Hf}$, had been saved from the original studies. In others, new grains were picked from the abraded zircon fractions and analyzed for both $\mathrm{U}-\mathrm{Pb}$ and $\mathrm{Hf}$ isotopes. $\mathrm{Zr}-\mathrm{Hf}$ column wash solutions were dried down and converted to weak $\mathrm{HNO}_{3}-\mathrm{HF}$ 


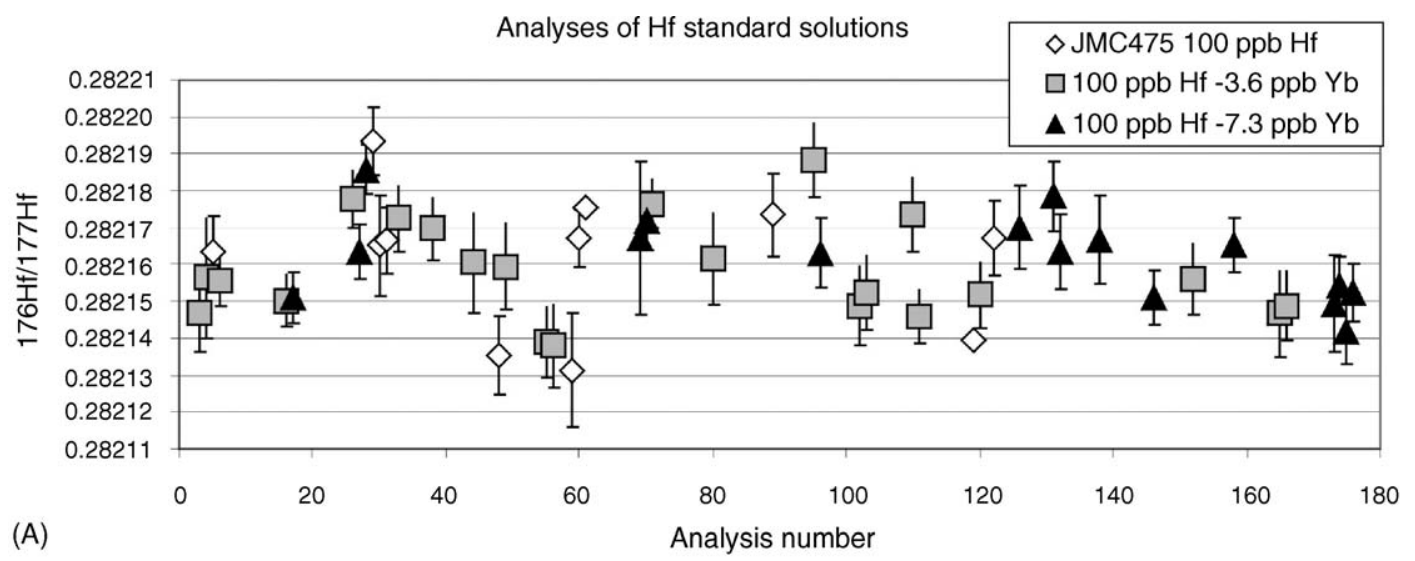

Averages of $\mathrm{Hf}-\mathrm{Yb}$ solution analyses

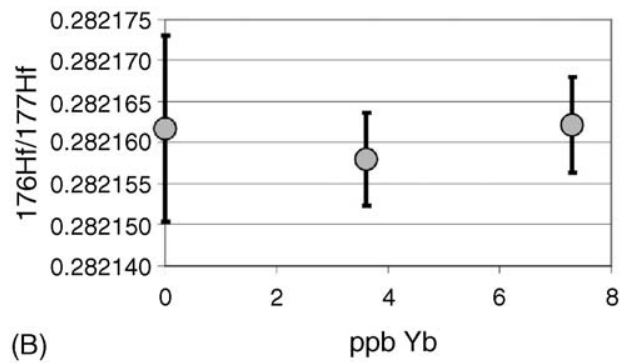

Analyses of 91500 zircon standard

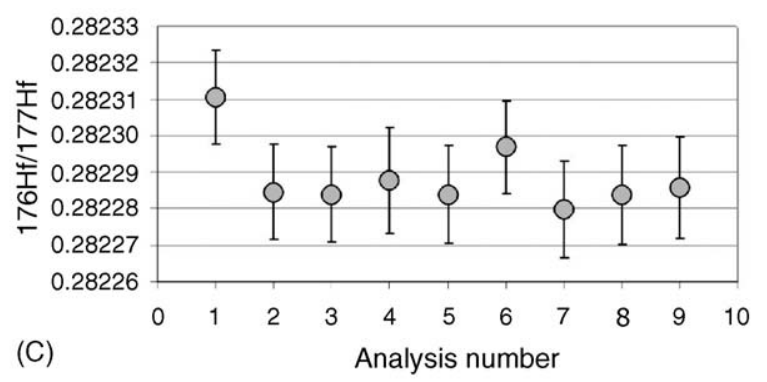

Fig. 2. (A) Measured values of $\mathrm{Hf}$ standards on the Plasma 54 instrument over the four-day period during which samples were measured. (B) Average values of ${ }^{176} \mathrm{Hf} /{ }^{177} \mathrm{Hf}$ for standard solutions containing different $\mathrm{Yb} / \mathrm{Hf}$ values. (C) $\varepsilon_{\mathrm{Hf}}$ values measured for zircon standard 91500 .

solutions, which were then aspirated directly into the plasma. Solutions were unspiked, and $\mathrm{Lu} / \mathrm{Hf}$ ratios were determined directly by measuring the ${ }^{175} \mathrm{Lu}$ and ${ }^{177} \mathrm{Hf}$ isotopes using the Plasma 54 at the NERC lab in Keyworth, U.K. (Nowell and Parrish, 2001). Corrections were made to $\mathrm{Hf}$ isotopes for $\mathrm{Lu}$ and $\mathrm{Yb}$ isobars by monitoring the ${ }^{173} \mathrm{Yb}$ and ${ }^{175} \mathrm{Lu}$ peaks. The major correction to the ${ }^{176} \mathrm{Hf}$ peak was for ${ }^{176} \mathrm{Yb}$. Sensitivity to the ${ }^{176} \mathrm{Yb}$ correction was monitored by analyzing $100 \mathrm{ppb}$ Hf solutions of JMC475. Different solutions containing $0 \mathrm{ppb} \mathrm{Yb}, 3.6 \mathrm{ppb} \mathrm{Yb}$ and $7.2 \mathrm{ppb} \mathrm{Yb}$ were used to test for bias to $\mathrm{Hf}$ isotopic measurements from the $\mathrm{Yb}$ correction (Fig. 2A). Averages of these separate standards are presented in Fig. 2B and show no systematic bias. Interference from REE-oxides was minimized by the use of a desolvating nebulizer. This is confirmed by the fact that ${ }^{180} \mathrm{Hf} /{ }^{177} \mathrm{Hf}$ and ${ }^{178} \mathrm{Hf} /{ }^{177} \mathrm{Hf}$ show no correlation with Lu/Hf. $R^{2}$ values are less then 0.02 for ${ }^{176} \mathrm{Lu} /{ }^{177} \mathrm{Hf}$ plotted against all other Hf isotopic ratios (all ratios reported in Table 2).
All errors are given at two standard deviations. Errors in $\varepsilon_{\mathrm{Hf}}$ are a combination of internal and external errors. Although internal standard errors for ${ }^{176} \mathrm{Hf} /{ }^{177} \mathrm{Hf}$ are generally less than $\pm 0.2 \varepsilon$ unit for all but the smallest samples, values of the JMC475 standard showed fluctuations outside of measurement errors during some periods (Fig. 2A). The series of measurements was divided into twelve periods over four days in which sample measurements could be bracketed by standards measured under relatively uniform instrumental operating conditions. The average of standard measurements during each of these periods was used to normalize sample results relative to an assumed ${ }^{176} \mathrm{Hf} /{ }^{177} \mathrm{Hf}$ value of 0.282160 for JMC475. These averages and the normalization factors are shown in Table 1 and are referenced to the Hf data in Table 2. The average of all 49 determinations of JMC475 is $0.282160 \pm 0.000028$ ( 2 sigma).

Previous Hf isotopic studies have shown that $\mathrm{Zr}-\mathrm{Hf}$ washes from the $\mathrm{U}-\mathrm{Pb}$ columns preserve the true $\mathrm{Lu} / \mathrm{Hf}$ 
Table 1

Normalization factors for analyses based on values measured for the JMC 475 standard

\begin{tabular}{rrlll}
\hline Norm. ref. & Run interval & JMC 475 measured & Errors 1 sig & Normalisation factors \\
\hline 1 & $3-17$ & 0.282154 & 0.000006 & 1.000022 \\
2 & $16-27$ & 0.282161 & 0.000013 & 0.999998 \\
3 & $31-44$ & 0.282167 & 0.000005 & 0.999974 \\
4 & $44-49$ & 0.282152 & 0.000014 & 1.000029 \\
5 & $49-55$ & 0.282149 & 0.000014 & 1.000038 \\
6 & $55-59$ & 0.282136 & 0.000004 & 1.000085 \\
7 & $60-89$ & 0.282170 & 0.000005 & 0.999963 \\
8 & $89-111$ & 0.282164 & 0.000016 & 0.999987 \\
9 & $119-120$ & 0.282146 & 0.000009 & 1.000051 \\
10 & $122-138$ & 0.282169 & 0.000006 & 0.999968 \\
11 & $138-146$ & 0.282159 & 0.000011 & 1.000004 \\
12 & $146-176$ & 0.282152 & 0.000007 & 1.000029 \\
\hline
\end{tabular}

Normalization Reference number is crossreferenced to Table 2. Run interval is the range of run numbers over which the normalization is applied (see Fig. 1A).

ratios of the samples (Amelin et al., 1999). However, inter-element fractionation in the plasma can amount to a deviation from the true value estimated at $3-15 \%$ by Nowell and Parrish (2001). Measurements of solutions from the 91500 zircon standard gave an average ${ }^{176} \mathrm{Lu} /{ }^{177} \mathrm{Hf}$ ratio of $0.000220 \pm 0.000052$. A consistent value of $0.000295 \pm 0.000030$ was measured for this standard by Amelin et al. (2000) and by Wiedenbeck et al. (1995). The Lu/Hf ratios are adjusted upward by a factor of $1.34 \pm 0.40$ to allow for this bias and its error is propagated into the errors in $\varepsilon_{\mathrm{Hf}}$. Normalized ${ }^{176} \mathrm{Hf} /{ }^{177} \mathrm{Hf}$ values measured on solutions of the 91500 zircon standard are presented in Fig. 2C. Most of these analyses agree within error except for one outlier. The average ${ }^{176} \mathrm{Hf} /{ }^{177} \mathrm{Hf}$ value of the nine measurements is $0.282298 \pm 20$, which becomes $0.282289 \pm 20$ after normalization to the nearest JMC475 standard measurements. The values measured by Wiedenbeck et al. (1995), Amelin et al. (2000) and Goolaerts et al. (2004) are $0.282302 \pm 8$, $0.282317 \pm 28$, and $0.282301 \pm 8$, respectively, after normalization to the average value of JMC475 found here. Our unnormalized value shows a better agreement, which suggests that short period instability may still bias the measurements to some extent.

\section{Results}

\subsection{Calculation of $\varepsilon_{H f}$ and model ages}

The resistance of Archean zircon to isotopic exchange of Hf was evaluated by Corfu and Noble
(1992), who found that zircon fractions from uniform aged populations with variable but moderate discordance $(<10 \%)$ have indistinguishable $\mathrm{Hf}$ isotopic ratios. This is to be expected since $\mathrm{Hf}$ is a relatively immobile element with a high concentration in zircon. In the present study, most of the Hf isotopic analysis were done on near-concordant zircon and are therefore likely to represent primary values.

Results of Hf isotopic measurements are given in Table 2. Except in the case of detrital and inherited zircons, most analyses were done on rocks with wellcharacterized ages based on multiple U-Pb zircon analyses. Previously unpublished $\mathrm{U}-\mathrm{Pb}$ results are given in Table 3. Rock samples are given sequential reference numbers in Table 2. Those in boldface are from previously unpublished projects and are located on the map in Fig. 1. References to previously published data sets are given in Table 2. Samples are grouped in the same order in Tables 2 and 3.

Calculated values of $\varepsilon_{\mathrm{Hf}}$ are plotted on Figs. 3-5 against age. For Archean samples, $\varepsilon_{\mathrm{Hf}}$ depends strongly on the value chosen for the ${ }^{176} \mathrm{Lu}$ decay constant because of its effect on the chondritic reference curve. In this work, we have adopted the value of $1.865 \pm 0.015 \times 10^{-5} \mathrm{~m} . \mathrm{y} .{ }^{-1}$ proposed by Scherer et al. (2001), based on U-Pb and $\mathrm{Lu}-\mathrm{Hf}$ dating of mineral phases in terrestrial rocks. Results published by Corfu and Noble (1992) and Corfu and Stott (1993b, 1996) are lowered by $1 \varepsilon_{\mathrm{Hf}}$ unit after adjustment for accepted values of ${ }^{176} \mathrm{Hf} /{ }^{177} \mathrm{Hf}(0.282772 \pm 29)$ and ${ }^{176} \mathrm{Lu} /{ }^{177} \mathrm{Hf}(0.0332 \pm 2)$ in chondrites (Blichert-Toft and Albarède, 1997), as well as the decay constant and 
Table 2

Lu-Hf isotopic data on zircons from the western Superior province

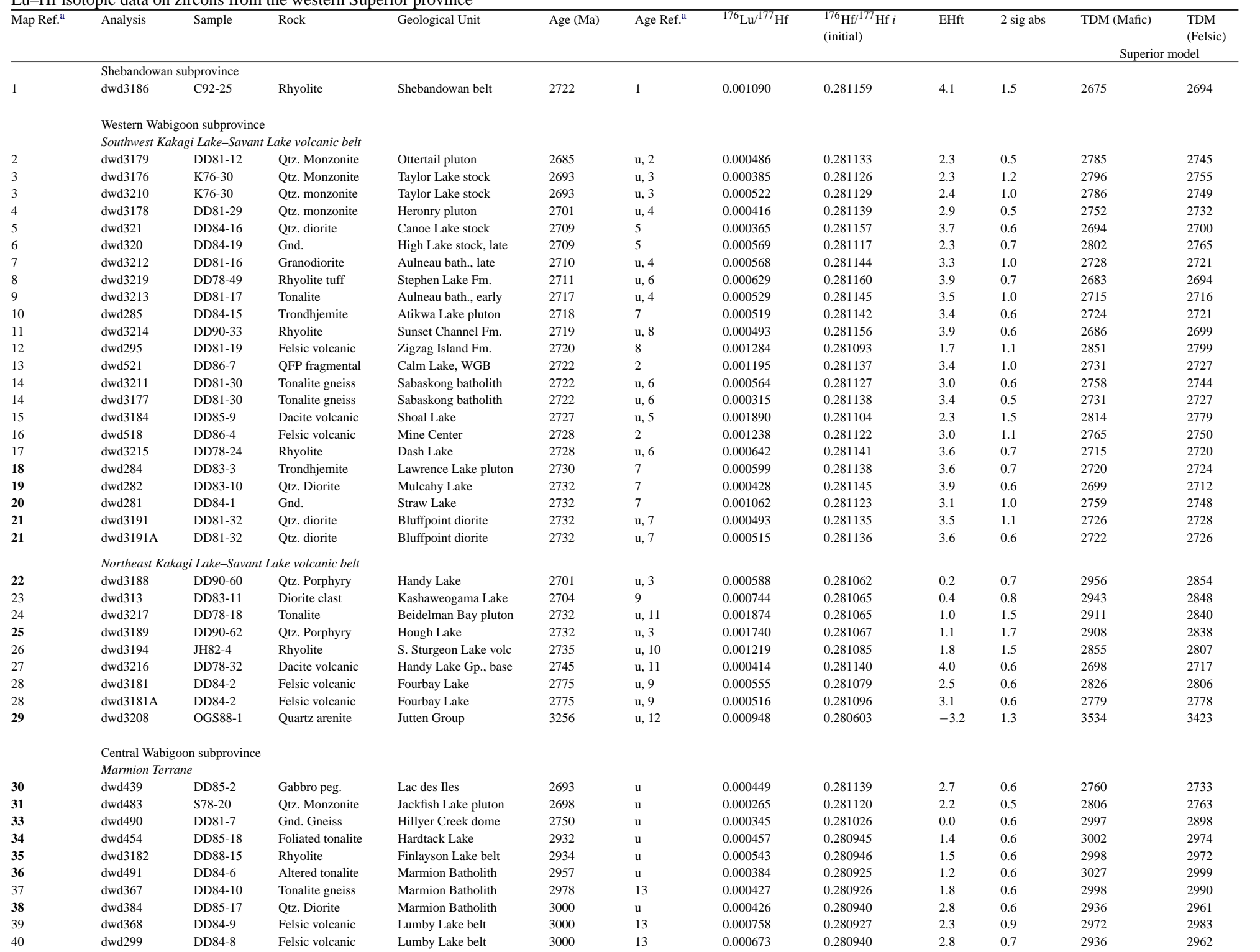




\begin{tabular}{|c|c|c|c|c|c|c|c|c|c|c|c|}
\hline \multicolumn{10}{|c|}{ North-central area } & & \\
\hline dwd3180 & DD96-10 & Qtz. monzonite & Roaring River complex & 2697 & u & 0.000213 & 0.281111 & 1.8 & 0.4 & 2831 & 2777 \\
\hline dwd3190 & 98KYT-37 & Rhyolite & N. Obonga Lake & 2707 & $\mathrm{u}, 14$ & 0.000456 & 0.281106 & 1.9 & 1.1 & 2832 & 2782 \\
\hline dwd 459 & DD85-28 & Felsic volcanic & Obonga Lake belt & 2726 & 14 & 0.000829 & 0.281114 & 2.6 & 1.0 & 2789 & 276 \\
\hline $\mathrm{dwd} 453$ & DD85-29 & Felsic volcanic & Obonga Lake belt & 2732 & 14 & 0.000706 & 0.281167 & 4.6 & 0.8 & 2642 & 2678 \\
\hline dwd3024 & DD96-11 & Foliated gnd. & Roaring River complex & 2739 & u & 0.000807 & 0.281074 & 1.5 & 1.0 & 2879 & 282 \\
\hline dwd475 & DD83-20 & Tonalite & Caribou Lake & 3075 & 9 & 0.000696 & 0.280739 & -2.6 & 1.3 & 3382 & 3266 \\
\hline \multicolumn{12}{|c|}{ Winnipeg River subprovince } \\
\hline dwd3193 & DD96-14 & Tonalite gneiss & Winnipeg River belt & 2721 & $\mathrm{u}, 15$ & 0.000445 & 0.280913 & -4.7 & 1.2 & 3329 & 308 \\
\hline $\mathrm{dwd} 474$ & DD81-18 & Tonalite gneiss & Tannis Lake & 3050 & 9 & 0.000388 & 0.280756 & -2.6 & 1.2 & 3367 & 3244 \\
\hline dwd3207 & C83-32HS & Gnd. gneiss & Cedar Lake pluton & 3188 & $\mathrm{u}, 16$ & 0.000901 & 0.280588 & -5.3 & 1.3 & 3650 & 3466 \\
\hline dwd3206 & C83-32HS & Gnd. gneiss & Cedar Lake pluton & 3225 & $\mathrm{u}, 16$ & 0.000832 & 0.280573 & -5.0 & 1.2 & 3647 & 3476 \\
\hline \multicolumn{12}{|c|}{ Bird River subprovince } \\
\hline dwd3185 & DD85-20 & Felsic porphyry & Separation Lake belt & 2723 & $\mathrm{u}$ & 0.000877 & 0.281012 & -1.1 & 0.8 & 3063 & 2927 \\
\hline \multicolumn{12}{|c|}{ English River subprovince } \\
\hline dwd3192 & C87-39 & Tonalite & English River belt & 2688 & $\mathrm{u}, 17$ & 0.000291 & 0.281003 & -2.2 & 1.1 & 3129 & 295 \\
\hline $\mathrm{dwd} 3218$ & C88-29 & Tonalite & Bluffy L. diorite & 2698 & u, 17 & 0.000344 & 0.281130 & 2.5 & 0.6 & 2778 & 274 \\
\hline dwd3187 & 97GRS700 & Rhyolite & Melchett Lake belt & 2726 & 18 & 0.000822 & 0.281147 & 3.8 & 0.7 & 2700 & 271 \\
\hline \multicolumn{12}{|c|}{ Sachigo subprovince } \\
\hline \multicolumn{12}{|c|}{ Igneous rocks } \\
\hline dwd304 & DD82-1 & Felsic volcanic & Oxford Lake group & 2709 & u & 0.000550 & 0.281122 & 2.5 & 0.7 & 2787 & 2756 \\
\hline $\mathrm{dwd} 3068$ & 88GRS501 & Dacite tuff & Ellard Lake & 2732 & 19 & 0.000485 & 0.281031 & -0.2 & 1.1 & 3002 & 289 \\
\hline $\mathrm{dwd} 458$ & DD85-26 & Tonalite gneiss & DeBliquey Lake & 2860 & 12 & 0.000438 & 0.281007 & 1.9 & 1.0 & 2920 & 2896 \\
\hline $\mathrm{dwd} 457$ & DD85-21 & Trondhjemite & N. Caribou Lake Bath. & 2870 & 12 & 0.000551 & 0.280987 & 1.5 & 0.9 & 2960 & 292 \\
\hline dwd3183 & DD87-20 & Rhyolite & Sandy Lake belt & 2945 & 19 & 0.000543 & 0.280962 & 2.3 & 0.6 & 2940 & 2942 \\
\hline $\mathrm{dwd} 449$ & DD85-23 & Felsic volcanic & Eyapamikama Lake & 2980 & 12 & 0.000492 & 0.280920 & 1.6 & 0.7 & 3011 & 2995 \\
\hline $\mathrm{dwd} 456$ & DD85-27 & Tonalite & Weagamow Bath. & 2990 & 12 & 0.000517 & 0.280911 & 1.5 & 0.9 & 3024 & 301 \\
\hline $\mathrm{dwd} 455$ & DD85-22 & Trond. Clast & Eyapamikama Lake & 3009 & 12 & 0.000856 & 0.280908 & 1.9 & 1.1 & 3012 & 301 \\
\hline \multicolumn{12}{|c|}{ Quartz arenite detrital zircons } \\
\hline dwd3071 & 88GRS114 & Qtzite clasts & Heaton Lake & 2853 & 19 & 0.000511 & 0.281005 & 1.7 & 0.9 & 2934 & 2902 \\
\hline dwd3090 & 88GRS408 & Pebbly arkose & Sandy Lake & 2905 & 19 & 0.000719 & 0.280997 & 2.6 & 2.2 & 2896 & 289 \\
\hline dwd3091 & 88GRS398 & Qtzite boulder & Sandy Lake & 2917 & 19 & 0.001432 & 0.281076 & 5.7 & 3.2 & 2671 & 276 \\
\hline dwd3072 & 88GRS114 & Qtzite clasts & Heaton Lake & 2980 & 19 & 0.000298 & 0.280953 & 2.8 & 0.8 & 2924 & 2946 \\
\hline $\mathrm{dwd} 3070$ & 88GRS114 & Qtzite clasts & Heaton Lake & 2981 & 19 & 0.000658 & 0.280933 & 2.1 & 0.9 & 2978 & 297 \\
\hline \multicolumn{12}{|c|}{ Quetico subprovince detrital zircons } \\
\hline dwd 3205 & Q98-10 & Sandstone & Quetico, Rainy Lake & 2700 & $\mathrm{u}$ & 0.000553 & 0.281085 & 1.0 & 1.2 & 2896 & 2818 \\
\hline dwd3204 & Q98-10 & Sandstone & Quetico, Rainy Lake & 2700 & $\mathrm{u}$ & 0.000618 & 0.280963 & -3.4 & 0.9 & 3220 & 301 ? \\
\hline dwd3201 & Q98-10 & Sandstone & Quetico, Rainy Lake & 2702 & $\mathrm{u}$ & 0.000496 & 0.281053 & -0.1 & 0.6 & 2978 & 2868 \\
\hline
\end{tabular}


Table 2 (Continued)

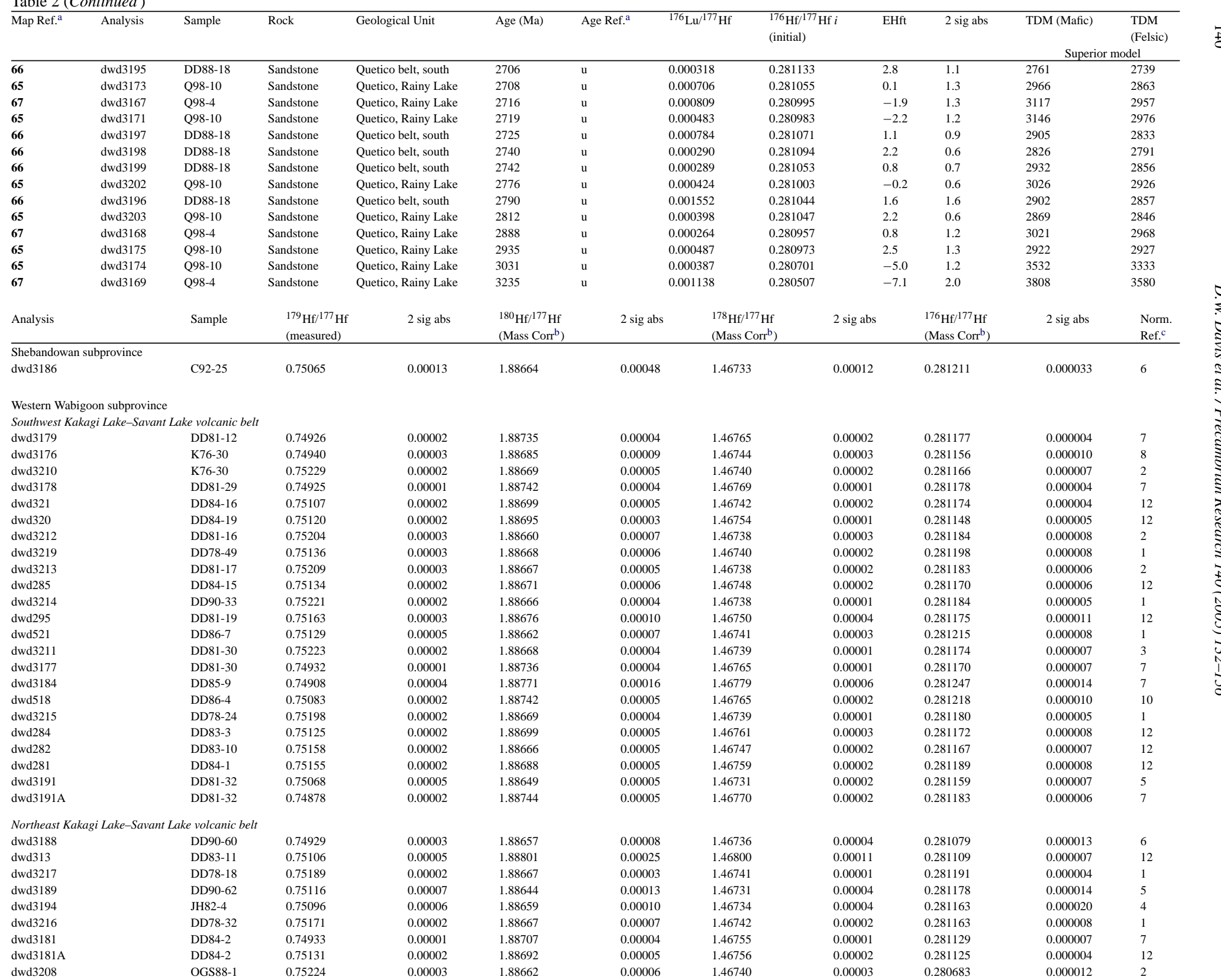


Central Wabigoon subprovince Marmion Terrane

$\begin{array}{ll}\mathrm{dwd} 439 & \text { DD85-2 } \\ \mathrm{dwd} 483 & \mathrm{~S}\end{array}$

$\begin{array}{ll}d w d 483 & \text { S78-20 } \\ d w d 490 & \text { DD81-7 }\end{array}$

dwd454 DD85-18

$\begin{array}{ll}\mathrm{dwd} 454 & \text { DD85-18 } \\ \mathrm{dwd} 3182 & \text { DD88-15 }\end{array}$

$\begin{array}{ll}\mathrm{dwd} 3182 & \text { DD88-15 } \\ \mathrm{dwd} 491 & \text { DD84-6 }\end{array}$

dwd367 DD84-10

dwd384 DD85-17

$\begin{array}{ll}\text { dwd368 } & \text { DD84-9 } \\ \text { dwd299 } & \text { DD84-8 }\end{array}$

North-central area

dwd3180 DD96-10

dwd3190 98KYT-37

dwd459 DD85-28

dwd453

dwd3024 DD96-11

dwd475 DD83-20

Winnipeg River subprovince

dwd3193 DD96-14

dwd474 DD81-18

dwd3207 C83-32HS

dwd3206 C83-32HS

Bird River subprovince

dwd3185 DD85-20

English River subprovince

dwd3192 C87-39

dwd3218 C88-29

dwd3187 $97 \mathrm{GRS} 700$

0.75042
0.75069

0.75030

0.74886

0.75015

0.75103

(n)

0.75133

0.74919

0.75079

0.75054

0.75015

0.74916

0.75023

0.74906

0.74782

0.74988

0.75170

0.74869

0.00004
0.00002
0.00002
0.00003
0.00002
0.00002
0.00003
0.00003
0.00003
0.00002

1.88703

1.88730
1.88742

1.88695

1.88705

1.88735
1.88691

1.88691

1.88746
1.887828

1.88698

$\begin{array}{ll}0.00001 & 1.88731\end{array}$

0.00004

0.00003

0.00004

0.00002

1.88649

1.88737

1.88734

1.88736

$0.00010 \quad 1.88656$

$\begin{array}{ll}0.00001 & 1.88716 \\ 0.00003 & 1.88665\end{array}$

0.00002

1.88668

0.00003

1.88795

0.00005

0.00003
0.00003

1.88653

1.88667
1.88664

\subsection{4}

0.00005
0.00005

0.00005

0.00006

0.00005

0.00007

0.00011

0.00007

0.00003

0.00003
0.00008

0.00005

0.00011

0.00008

0.00018

0.00018
0.00003

0.00003
0.00008

0.00005

0.00008

0.00011

0.00020

0.00007

1.46746

1.46762
1.46767

1.46745

1.46755

1.46761
1.46742

1.46765

1.467788

1.46764

1.46730

1.46764

1.46781
1.46765
1.46766

1.46766

1.46728
1.46758

1.46758
1.46738

1.46739

Sachigo subprovince

Igneous rocks

dwd304 DD82-1

0.75138

0.74934
0.75080

0.75080
0.75058

0.75058
0.74890

0.74981

0.75044

0.75084

0.00003

1.88692

1.88757

1.88732

1.88720

1.88721

1.88719
1.88721

0.0000

0.00003

0.0000

1.88717

Quartz arenite detrital zircon

dwd3071 88GRS114

dwd3090 88GRS408

dwd3091 88GRS398

dwd3072 88GRS114

0.75011

0.74780

0.74870

0.74966

1.88763

1.88797

1.88784
1.88725

0.0000

0.0000

1.88725
1.88721

Quetico subprovince detrital zircons

dwd 3205

Q98-10

0.75241

0.00003

1.88673

0.00008

0.00012

0.00007

0.00003
0.00007

0.00007

0.00008

0.00004
0.00003

1.46793

1.46733

1.46733
1.46738
1.46737

0.00007

0.00025

0.00033

0.00008

1.46785
1.46777

1.46777

0.00009

1.46758

0.00009
0.00008

1.46743
1.46737

1.46753
1.46774

1.46774
1.46762

1.46757
1.46758

1.46754

1.46757

1.8866

1.46737

$\begin{array}{ll}0.00001 & 0.281162 \\ 0.00002 & 0.281147 \\ 0.00002 & 0.281059 \\ 0.00002 & 0.280971 \\ 0.00002 & 0.280997 \\ 0.00002 & 0.280963 \\ 0.00003 & 0.280951 \\ 0.00005 & 0.280965 \\ 0.00003 & 0.280977 \\ 0.00002 & 0.280984 \\ & \\ 0.00002 & 0.281136 \\ 0.00003 & 0.281127 \\ 0.00002 & 0.281171 \\ 0.00005 & 0.281208 \\ 0.00003 & 0.281117 \\ 0.00002 & 0.280798 \\ & \\ 0.00004 & 0.280933 \\ 0.00001 & 0.280790 \\ 0.00003 & 0.280663 \\ 0.00002 & 0.280643 \\ & \\ 0.00003 & 0.281084 \\ & \\ 0.00003 & 0.281012 \\ 0.00002 & 0.281148 \\ 0.00002 & 0.281181 \\ & \\ 0.000 & \\ 0.00005 & 0.281152 \\ 0.00003 & 0.281051 \\ 0.00001 & 0.281038 \\ 0.00003 & 0.281014 \\ 0.00003 & 0.280950 \\ 0.00002 & 0.280950 \\ 0.00001 & 0.280973 \\ 0.000003 & \\ 0.00008 & 0.281028 \\ 0.00009 & 0.281054 \\ 0.00005 & 0.281187 \\ 0.00003 & 0.280969 \\ & \\ 0.00004 & 0.281124 \\ & \\ & \end{array}$

\begin{tabular}{|c|c|}
\hline 0.000006 & 12 \\
\hline 0.000008 & 10 \\
\hline 0.000010 & 10 \\
\hline 0.000004 & 12 \\
\hline 0.000008 & 7 \\
\hline 0.000007 & 10 \\
\hline 0.000007 & 12 \\
\hline 0.000004 & 12 \\
\hline 0.000010 & 12 \\
\hline 0.000005 & 12 \\
\hline 0.000004 & 7 \\
\hline 0.000008 & 5 \\
\hline 0.000008 & 11 \\
\hline 0.000007 & 12 \\
\hline 0.000014 & 9 \\
\hline 0.000009 & 8 \\
\hline 0.000014 & 5 \\
\hline 0.000007 & 8 \\
\hline 0.000010 & 2 \\
\hline 0.000007 & 2 \\
\hline 0.000008 & 7 \\
\hline 0.000008 & 5 \\
\hline 0.000008 & 1 \\
\hline 0.000008 & 6 \\
\hline 0.000010 & 12 \\
\hline 0.000022 & 9 \\
\hline 0.000013 & 11 \\
\hline 0.000006 & 11 \\
\hline 0.000007 & 7 \\
\hline 0.000010 & 12 \\
\hline 0.000006 & 11 \\
\hline 0.000005 & 11 \\
\hline 0.000013 & 9 \\
\hline 0.000049 & 8 \\
\hline 0.000079 & 8 \\
\hline 0.000015 & 9 \\
\hline 0.000010 & 9 \\
\hline 0.000017 & 2 \\
\hline
\end{tabular}




\begin{tabular}{|c|c|c|c|c|c|c|c|c|c|c|}
\hline Analysis & Sample & $\begin{array}{l}{ }^{179} \mathrm{Hf} / 177 \mathrm{Hf} \\
\text { (measured) }\end{array}$ & 2 sig abs & $\begin{array}{l}{ }^{180} \mathrm{Hf} / 177 \mathrm{Hf} \\
\left(\text { Mass Corr }{ }^{\mathrm{b}}\right)\end{array}$ & 2 sig abs & $\begin{array}{l}{ }^{178} \mathrm{Hf} / 177 \mathrm{Hf} \\
\left(\text { Mass Corr }{ }^{\mathrm{b}}\right)\end{array}$ & 2 sig abs & $\begin{array}{l}{ }^{176} \mathrm{Hf} / /^{177} \mathrm{Hf} \\
\left(\text { Mass Corr }{ }^{\mathrm{b}}\right)\end{array}$ & 2 sig abs & $\begin{array}{l}\text { Norm. } \\
\text { Ref. }\end{array}$ \\
\hline dwd3201 & Q98-10 & 0.75040 & 0.00007 & 1.88650 & 0.00008 & 1.46734 & 0.00002 & 0.281095 & 0.000007 & 3 \\
\hline dwd3195 & DD88-18 & 0.75111 & 0.00004 & 1.88654 & 0.00006 & 1.46735 & 0.00002 & 0.281147 & 0.000011 & 4 \\
\hline dwd3173 & Q98-10 & 0.74886 & 0.00003 & 1.88728 & 0.00008 & 1.46765 & 0.00003 & 0.281108 & 0.000013 & 8 \\
\hline dwd3167 & Q98-4 & 0.74868 & 0.00002 & 1.88731 & 0.00006 & 1.46764 & 0.00002 & 0.281055 & 0.000008 & 8 \\
\hline dwd3171 & Q98-10 & 0.74874 & 0.00003 & 1.88720 & 0.00005 & 1.46759 & 0.00002 & 0.281020 & 0.000008 & 8 \\
\hline dwd3197 & DD88-18 & 0.75109 & 0.00005 & 1.88642 & 0.00009 & 1.46734 & 0.00003 & 0.281133 & 0.000016 & 3 \\
\hline dwd3198 & DD88-18 & 0.75099 & 0.00005 & 1.88658 & 0.00008 & 1.46736 & 0.00003 & 0.281122 & 0.000013 & 3 \\
\hline dwd3199 & DD88-18 & 0.75079 & 0.00004 & 1.88667 & 0.00010 & 1.46734 & 0.00004 & 0.281081 & 0.000016 & 3 \\
\hline dwd3202 & Q98-10 & 0.75001 & 0.00003 & 1.88653 & 0.00007 & 1.46731 & 0.00002 & 0.281041 & 0.000011 & 3 \\
\hline dwd3196 & DD88-18 & 0.75092 & 0.00005 & 1.88660 & 0.00009 & 1.46733 & 0.00004 & 0.281147 & 0.000015 & 4 \\
\hline dwd3203 & Q98-10 & 0.74983 & 0.00003 & 1.88648 & 0.00008 & 1.46729 & 0.00003 & 0.281083 & 0.000011 & 3 \\
\hline dwd3168 & Q98-4 & 0.74874 & 0.00003 & 1.88723 & 0.00007 & 1.46758 & 0.00002 & 0.280980 & 0.000012 & 8 \\
\hline $\mathrm{dwd} 3175$ & Q98-10 & 0.74939 & 0.00003 & 1.88749 & 0.00009 & 1.46775 & 0.00004 & 0.281014 & 0.000015 & 8 \\
\hline $\mathrm{dwd} 3174$ & Q98-10 & 0.74930 & 0.00003 & 1.88731 & 0.00007 & 1.46759 & 0.00002 & 0.280735 & 0.000012 & 8 \\
\hline $\mathrm{dwd} 3169$ & Q98-4 & 0.74856 & 0.00004 & 1.88731 & 0.00011 & 1.46754 & 0.00005 & 0.280606 & 0.000037 & 8 \\
\hline
\end{tabular}

${ }^{a}$ Map Ref.: Sample numbers in bold are located on Fig. 1. Others are located in their referenced publications. Age Ref.: u—unpublished analysis, U-Pb data are given in Table 3. More extensive U-Pb data are presented in numbered references: 1. Corfu and Stott, 1998; 2. Davis et al., 1989; 3. Davis, 1995; 4. Davis and Edwards, 1986; 5. Davis and Smith, 1991; 6. Davis and Edwards, 1982; 7. Davis and Edwards, 1985; 8. Ayer and Davis, 1997; 9. Davis et al., 1988; 10. Davis et al., 1985; 11. Davis and Trowell, 1982; 12. Davis and Moore, 1991; 13. Davis and Jackson, 1988; 14. Tomlinson et al., 2002; 15. Cruden et al., 1997; 16. Corfu, 1988; 17. Corfu et al., 1995; 18. Davis, 1999; 19. Davis and Stott, 2002.

${ }^{\mathrm{b}}$ Mass Corr: Corrected for exponential mass fractionation assuming true ${ }^{179} \mathrm{Hf} /{ }^{177} \mathrm{Hf}=0.7325$.

${ }^{\mathrm{c}}$ Norm. Ref.: Normalization values based on JMC475 are given in Table 1.
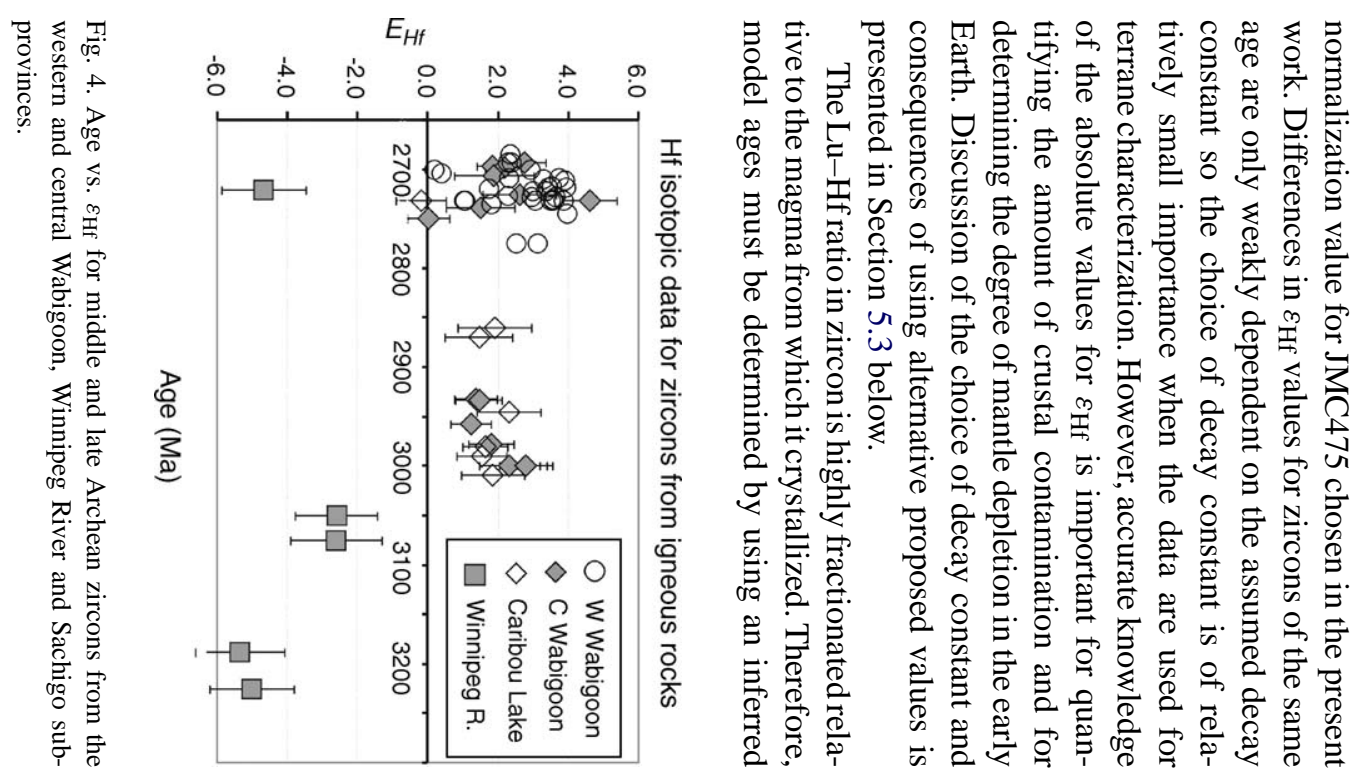

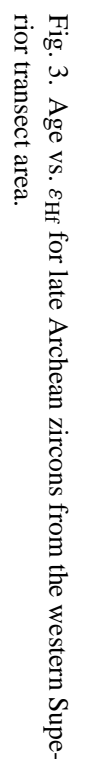
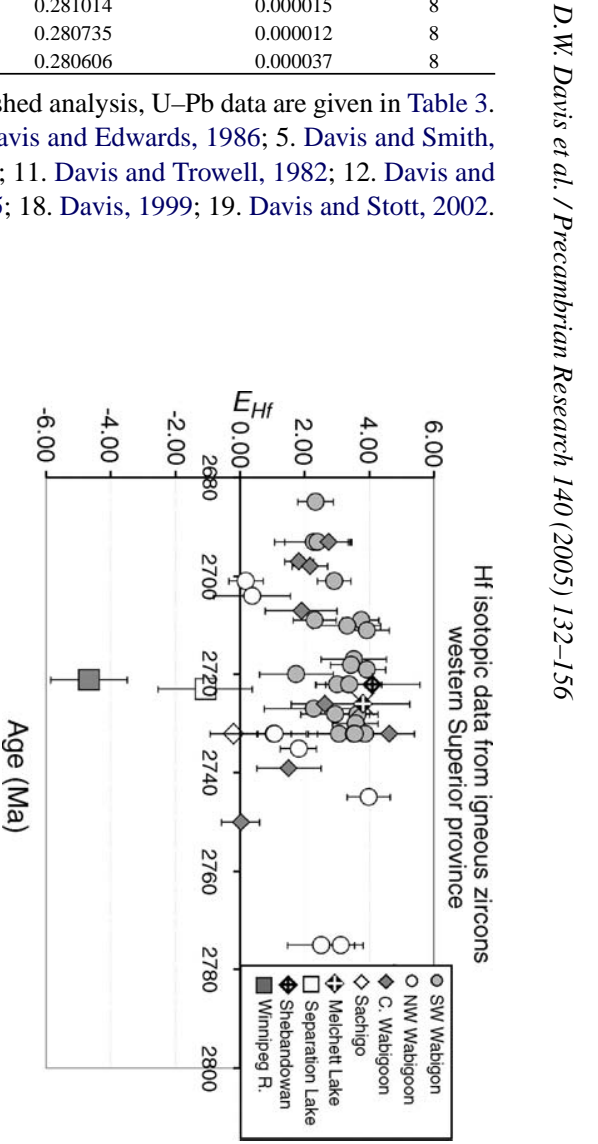
Table 3

$\mathrm{U}-\mathrm{Pb}$ isotopic data on zircon analyzed for $\mathrm{Hf}$ isotopes from the western Superior province

\begin{tabular}{|c|c|c|c|c|c|c|c|c|c|c|c|c|c|c|}
\hline Analysis no. & Sample & Wt. (mg) & $\mathrm{U}(\mathrm{ppm})$ & $\mathrm{Th} / \mathrm{U}$ & $\begin{array}{l}\text { PbCom } \\
(\mathrm{pg})\end{array}$ & ${ }^{207} \mathrm{~Pb} /{ }^{204} \mathrm{~Pb}$ & ${ }^{206} \mathrm{~Pb} /{ }^{238} \mathrm{U}$ & $2 \mathrm{sig}$ & ${ }^{207} \mathrm{~Pb} /{ }^{235} \mathrm{U}$ & $2 \mathrm{sig}$ & ${ }^{207} \mathrm{~Pb} /{ }^{206} \mathrm{~Pb}$ & $2 \mathrm{sig}$ & Disc. $(\%)$ & $\begin{array}{l}\text { Correl } \\
\text { coeff. }\end{array}$ \\
\hline \multicolumn{15}{|c|}{ 1. Shebandowan subprovince } \\
\hline $\mathrm{dwd} 3186$ & $\mathrm{C} 92-25$ & 0.017 & 63 & 0.50 & 0.7 & 10405 & 0.5274 & 0.0016 & 13.645 & 0.045 & 2721.7 & 1.7 & -0.4 & 0.9517 \\
\hline \multicolumn{15}{|c|}{ Western Wabigoon subprovince } \\
\hline \multicolumn{15}{|c|}{ 2. Southwest Kakagi Lake-Savant Lake volcanic belt } \\
\hline dwd3179 & DD81-12 & 0.048 & 72 & 0.89 & 3.6 & 5789 & 0.5124 & 0.0014 & 12.970 & 0.038 & 2685.3 & 1.7 & 0.8 & 0.9328 \\
\hline $\mathrm{dwd} 3176$ & K76-30 & 0.007 & 224 & 0.81 & 0.7 & 13722 & 0.5166 & 0.0013 & 13.135 & 0.035 & 2692.8 & 1.8 & 0.4 & 0.9122 \\
\hline dwd 3210 & K76-30 & 0.030 & 262 & 0.82 & 24.5 & 1712 & 0.4942 & 0.0025 & 12.553 & 0.065 & 2691.4 & 2.1 & 4.6 & 0.9705 \\
\hline $\mathrm{dwd} 3178$ & DD81-29 & 0.019 & 135 & 0.69 & 2.3 & 6926 & 0.5107 & 0.0025 & 13.051 & 0.066 & 2701.4 & 1.4 & 1.9 & 0.9858 \\
\hline $\mathrm{dwd} 3212$ & DD81-16 & 0.005 & 213 & 0.50 & 3.4 & 1955 & 0.5146 & 0.0012 & 13.116 & 0.032 & 2696.9 & 2.2 & 0.9 & 0.8394 \\
\hline dwd3219 & DD78-49 & 0.015 & 75 & 0.77 & 1.7 & 4232 & 0.5232 & 0.0012 & 13.446 & 0.034 & 2710.5 & 2.0 & -0.1 & 0.8795 \\
\hline dwd3213 & DD81-17 & 0.011 & 79 & 0.38 & 4.0 & 1363 & 0.5217 & 0.0013 & 13.449 & 0.038 & 2715.7 & 1.8 & 0.4 & 0.9228 \\
\hline dwd3214 & DD90-33 & 0.030 & 57 & 0.56 & 1.5 & 7226 & 0.5205 & 0.0018 & 13.427 & 0.048 & 2716.7 & 1.7 & 0.7 & 0.9578 \\
\hline dwd3211 & DD81-30 & 0.026 & 169 & 0.21 & 40.2 & 476 & 0.4022 & 0.0021 & 10.335 & 0.055 & 2710.3 & 2.5 & 23.0 & 0.9603 \\
\hline dwd3177 & DD81-30 & 0.016 & 63 & 0.23 & 9.8 & 655 & 0.5198 & 0.0026 & 13.455 & 0.071 & 2722.3 & 2.3 & 1.1 & 0.9655 \\
\hline dwd3184 & DD85-9 & 0.011 & 197 & 0.50 & 2.5 & 5520 & 0.5187 & 0.0039 & 13.464 & 0.094 & 2727.0 & 5.2 & 1.5 & 0.9089 \\
\hline dwd3215 & DD78-24 & 0.027 & 86 & 0.35 & 195.6 & 90.71 & 0.5257 & 0.0016 & 13.641 & 0.118 & 2726.4 & 10.9 & 0.1 & 0.7693 \\
\hline dwd3191 & DD81-32 & 0.062 & 43 & 0.64 & 2.1 & 8090 & 0.5271 & 0.0013 & 13.724 & 0.038 & 2732.1 & 1.7 & 0.1 & 0.9300 \\
\hline \multicolumn{15}{|c|}{ 3. Northeast Kakagi Lake-Savant Lake volcanic belt } \\
\hline dwd 3188 & DD90-60 & 0.010 & 87 & 0.67 & 3.1 & 1763 & 0.5134 & 0.0030 & 13.120 & 0.079 & 2701.2 & 1.9 & 1.4 & 0.9820 \\
\hline dwd 3217 & DD78-18 & 0.054 & 384 & 0.85 & 615.5 & 222 & 0.5132 & 0.0025 & 13.179 & 0.072 & 2709.5 & 5.6 & 1.8 & 0.7897 \\
\hline dwd3189 & DD90-62 & 0.007 & 221 & 0.65 & 2.9 & 3436 & 0.5259 & 0.0017 & 13.690 & 0.044 & 2731.8 & 2.6 & 0.3 & 0.8800 \\
\hline dwd3194 & JH82-4 & 0.018 & 13 & 0.51 & 0.8 & 1979 & 0.5242 & 0.0019 & 13.660 & 0.053 & 2733.3 & 2.1 & 0.7 & 0.9445 \\
\hline $\mathrm{dwd} 3216$ & DD78-32 & 0.035 & 100 & 0.39 & 388.2 & 70.21 & 0.4953 & 0.0025 & 12.979 & 0.158 & 2742.7 & 15.3 & 6.6 & 0.7133 \\
\hline dwd3181 & DD84-2 & 0.028 & 97 & 0.52 & 0.7 & 13367 & 0.2846 & 0.0057 & 7.605 & 0.153 & 2774.9 & 2.0 & 47.1 & 0.9982 \\
\hline dwd3208 & OGS88-1 & 0.003 & 74 & 0.98 & 2.4 & 1039 & 0.6533 & 0.0020 & 23.553 & 0.078 & 3255.7 & 2.2 & 0.6 & 0.9111 \\
\hline \multicolumn{15}{|c|}{ Central Wabigoon subprovince } \\
\hline \multicolumn{15}{|c|}{ 4. Marmion Terrane } \\
\hline dwd439 & DD85-2 & 0.068 & 148 & 1.00 & 19.2 & 3207 & 0.51537 & 0.0018 & 13.0782 & 0.047 & 2689.7 & 2.1 & 0.5 & 0.9336 \\
\hline dwd483 & S78-20 & 0.100 & 102 & 0.88 & 20.7 & 3043 & 0.51727 & 0.0018 & 13.1748 & 0.047 & 2695.8 & 2.1 & 0.4 & 0.9342 \\
\hline dwd490 & DD81-7 & 0.015 & 56 & 0.34 & 5.35 & 1036 & 0.53191 & 0.0026 & 14.0212 & 0.071 & 2752.3 & 2.3 & 0.1 & 0.9592 \\
\hline dwd454 & DD85-18 & 0.042 & 110 & 0.44 & 810.3 & 60.23 & 0.57185 & 0.0028 & 16.8272 & 0.243 & 2931.7 & 17.2 & 0.7 & 0.8462 \\
\hline dwd3182 & DD88-15 & 0.016 & 70 & 0.47 & 0.7 & 12019 & 0.5743 & 0.0022 & 16.924 & 0.069 & 2934.0 & 1.5 & 0.4 & 0.9738 \\
\hline dwd491 & DD84-6 & 0.027 & 34 & 0.58 & 6.35 & 1180 & 0.57747 & 0.0028 & 17.2532 & 0.087 & 2956.2 & 2.2 & 0.8 & 0.9622 \\
\hline $\mathrm{dwd} 384$ & DD85-17 & 0.240 & 52 & 0.46 & 19.45 & 5351 & 0.58756 & 0.0035 & 18.0129 & 0.110 & 2997.8 & 2.1 & 0.8 & 0.9774 \\
\hline \multicolumn{15}{|c|}{ 5. North-central area } \\
\hline dwd 3180 & DD96-10 & 0.075 & 124 & 1.09 & 4.0 & 14276 & 0.5184 & 0.0012 & 13.213 & 0.035 & 2696.8 & 1.5 & 0.2 & 0.9433 \\
\hline dwd3190 & 98KYT-37 & 0.008 & 133 & 0.72 & 8.7 & 770 & 0.5191 & 0.0017 & 13.310 & 0.046 & 2706.8 & 2.2 & 0.5 & 0.9207 \\
\hline dwd3024 & DD96-11 & 0.010 & 28 & 0.71 & 1.3 & 1410 & 0.5286 & 0.0018 & 13.819 & 0.051 & 2738.9 & 2.0 & 0.2 & 0.9448 \\
\hline \multicolumn{15}{|c|}{ 6. Winnipeg River subprovince } \\
\hline dwd 3193 & DD96-14 & 0.007 & 224 & 0.49 & 2.7 & 3585 & 0.5126 & 0.0016 & 13.252 & 0.043 & 2720.5 & 2.2 & 2.4 & 0.9150 \\
\hline dwd3207 & $\mathrm{C} 83-32 \mathrm{HS}$ & 0.004 & 310 & 0.49 & 1.5 & 8579 & 0.6346 & 0.0022 & 21.924 & 0.080 & 3188.4 & 1.5 & 0.8 & 0.9672 \\
\hline dwd3206 & $\mathrm{C} 83-32 \mathrm{HS}$ & 0.007 & 380 & 0.56 & 5.4 & 4843 & 0.5927 & 0.0023 & 20.961 & 0.083 & 3225.3 & 2.2 & 8.7 & 0.9388 \\
\hline \multicolumn{15}{|c|}{ 7. Bird River subprovince } \\
\hline $\mathrm{dwd} 3185$ & DD95-20 & 0.013 & 331 & 0.53 & 1.3 & 21839 & 0.5253 & 0.0012 & 13.600 & 0.035 & 2722.7 & 1.6 & 0.0 & 0.9256 \\
\hline \multicolumn{15}{|c|}{ 8. English River subprovince } \\
\hline dwd3192 & C87-39 & 0.017 & 267 & 0.62 & 6.4 & 4319 & 0.5108 & 0.0013 & 12.949 & 0.036 & 2688.0 & 1.7 & 1.3 & 0.9284 \\
\hline $\mathrm{dwd} 3218$ & C88-29 & 0.010 & 397 & 0.83 & 3.8 & 6486 & 0.5201 & 0.0039 & 13.290 & 0.100 & 2701.2 & 2.9 & 0.1 & 0.9721 \\
\hline dwd3187 & 97GRS700 & 0.022 & 72 & 0.55 & 0.8 & 13045 & 0.5249 & 0.0030 & 13.613 & 0.080 & 2725.6 & 1.7 & 0.3 & 0.9843 \\
\hline \multicolumn{15}{|c|}{ Sachigo subprovince } \\
\hline \multicolumn{15}{|c|}{ 9. Igneous rocks } \\
\hline dwd304 & DD82-1 & 0.085 & 151 & 0.47 & 196.3 & 371 & 0.5201 & 0.0021 & 13.322 & 0.052 & 2705.0 & 4.3 & 0.2 & 0.7844 \\
\hline dwd3068 & 88GRS501 & 0.010 & 22 & 0.79 & 0.9 & 1544 & 0.5251 & 0.0016 & 13.672 & 0.044 & 2732.0 & 2.1 & 0.5 & 0.9208 \\
\hline dwd458 & DD85-26 & 0.004 & 287 & 0.25 & 5.5 & 1527 & 0.5553 & 0.0028 & 15.589 & 0.078 & 2855.3 & 2.7 & 0.4 & 0.9441 \\
\hline dwd457 & DD85-21 & 0.023 & 72 & 0.42 & 5.6 & 2184 & 0.5585 & 0.0020 & 15.810 & 0.057 & 2868.9 & 2.7 & 0.4 & 0.8961 \\
\hline dwd 3183 & DD87-20 & 0.010 & 289 & 0.58 & 1.4 & 16218 & 0.5768 & 0.0042 & 17.114 & 0.120 & 2945.0 & 3.9 & 0.4 & 0.9428 \\
\hline dwd449 & DD85-23 & 0.041 & 97 & 0.91 & 15.0 & 2205 & 0.5866 & 0.0020 & 17.791 & 0.064 & 2980.5 & 2.1 & 0.2 & 0.9309 \\
\hline dwd456 & DD85-27 & 0.035 & 159 & 0.68 & 8.0 & 5803 & 0.5873 & 0.0020 & 17.911 & 0.064 & 2989.3 & 2.1 & 0.5 & 0.9344 \\
\hline dwd455 & DD85-22 & 0.048 & 139 & 0.54 & 8.7 & 6011 & 0.5628 & 0.0020 & 16.834 & 0.060 & 2958.2 & 2.1 & 3.4 & 0.9347 \\
\hline
\end{tabular}


Table 3 (Continued)

\begin{tabular}{|c|c|c|c|c|c|c|c|c|c|c|c|c|c|c|}
\hline Analysis no. & Sample & Wt. (mg) & $\mathrm{U}(\mathrm{ppm})$ & $\mathrm{Th} / \mathrm{U}$ & $\begin{array}{l}\text { PbCom } \\
(\mathrm{pg})\end{array}$ & ${ }^{207} \mathrm{~Pb} /{ }^{204} \mathrm{~Pb}$ & ${ }^{206} \mathrm{~Pb} /{ }^{238} \mathrm{U}$ & $2 \operatorname{sig}$ & ${ }^{207} \mathrm{~Pb} /{ }^{235} \mathrm{U}$ & $2 \mathrm{sig}$ & ${ }^{207} \mathrm{~Pb} / 206 \mathrm{~Pb}$ & $2 \mathrm{sig}$ & Disc. (\%) & $\begin{array}{l}\text { Correl. } \\
\text { coeff. }\end{array}$ \\
\hline \multicolumn{15}{|c|}{ 10. Quartz arenite detrital zircons } \\
\hline dwd3071 & 88 GRS 114 & 0.020 & 12 & 0.49 & 1.8 & 1014 & 0.5530 & 0.0026 & 15.504 & 0.075 & 2853.1 & 1.9 & 0.7 & 0.9697 \\
\hline dwd3090 & 88GRS408 & 0.008 & 7 & 1.05 & 1.2 & 380 & 0.5559 & 0.0025 & 16.086 & 0.108 & 2904.7 & 6.3 & 2.3 & 0.8314 \\
\hline dwd3091 & 88 GRS398 & 0.008 & 13 & 0.49 & 0.5 & 1448 & 0.5653 & 0.0024 & 16.488 & 0.065 & 2917.4 & 4.4 & 1.2 & 0.7783 \\
\hline dwd3072 & 88 GRS 114 & 0.010 & 73 & 1.01 & 0.6 & 10146 & 0.5867 & 0.0020 & 17.791 & 0.066 & 2980.1 & 1.5 & 0.2 & 0.9681 \\
\hline dwd 3070 & $88 \mathrm{GRS} 114$ & 0.020 & 63 & 0.74 & 6.8 & 1543 & 0.5857 & 0.0028 & 17.773 & 0.087 & 2981.2 & 1.7 & 0.4 & 0.9773 \\
\hline \multicolumn{15}{|c|}{ 11. Quetico subprovince detrital zircons } \\
\hline $\mathrm{dwd} 3205$ & Q-10 & 0.001 & 318 & 0.90 & 0.6 & 3181 & 0.5155 & 0.0016 & 13.166 & 0.043 & 2700.3 & 2.1 & 0.9 & 0.9210 \\
\hline dwd3204 & Q-10 & 0.002 & 182 & 0.83 & 0.5 & 4711 & 0.5161 & 0.0018 & 13.180 & 0.048 & 2700.1 & 1.8 & 0.8 & 0.9515 \\
\hline $\mathrm{dwd} 3201$ & Q-10 & 0.009 & 152 & 1.11 & 3.2 & 2642 & 0.5129 & 0.0016 & 13.110 & 0.043 & 2701.5 & 1.9 & 1.5 & 0.9390 \\
\hline dwd3195 & DD88-18 & 0.005 & 64 & 1.21 & 1.0 & 2112 & 0.5200 & 0.0016 & 13.323 & 0.043 & 2705.5 & 2.1 & 0.3 & 0.9225 \\
\hline $\mathrm{dwd} 3173$ & Q10-98 & 0.005 & 49 & 0.56 & 4.3 & 369 & 0.5159 & 0.0016 & 13.241 & 0.049 & 2708.3 & 3.1 & 1.2 & 0.8671 \\
\hline $\mathrm{dwd} 3167$ & Q4-98 & 0.009 & 27 & 0.95 & 1.2 & 1257 & 0.5246 & 0.0022 & 13.526 & 0.060 & 2715.8 & 1.9 & -0.1 & 0.9653 \\
\hline dwd3171 & Q10-98 & 0.020 & 56 & 0.91 & 0.6 & 11207 & 0.5223 & 0.0024 & 13.490 & 0.064 & 2718.9 & 2.3 & 0.5 & 0.9572 \\
\hline dwd3197 & DD88-18 & 0.003 & 45 & 0.62 & 1.0 & 849 & 0.5217 & 0.0032 & 13.524 & 0.084 & 2724.7 & 3.4 & 0.8 & 0.9446 \\
\hline dwd3198 & DD88-18 & 0.003 & 59 & 0.41 & 1.4 & 848 & 0.5269 & 0.0029 & 13.783 & 0.107 & 2739.6 & 8.3 & 0.5 & 0.7604 \\
\hline dwd3199 & DD88-18 & 0.003 & 49 & 0.37 & 1.0 & 951 & 0.5278 & 0.0027 & 13.829 & 0.069 & 2742.4 & 3.5 & 0.5 & 0.9097 \\
\hline dwd3202 & Q-10 & 0.005 & 120 & 0.74 & 0.6 & 6889 & 0.5367 & 0.0018 & 14.356 & 0.051 & 2776.4 & 1.5 & 0.3 & 0.9654 \\
\hline dwd3196 & DD88-18 & 0.004 & 25 & 0.62 & 0.9 & 770 & 0.5386 & 0.0023 & 14.531 & 0.068 & 2790.5 & 2.6 & 0.6 & 0.9408 \\
\hline dwd 3203 & Q-10 & 0.003 & 84 & 0.64 & 0.5 & 3600 & 0.5435 & 0.0019 & 14.849 & 0.050 & 2810.9 & 2.7 & 0.6 & 0.8835 \\
\hline dwd3168 & Q4-98 & 0.006 & 68 & 0.38 & 1.9 & 1667 & 0.5626 & 0.0018 & 16.117 & 0.055 & 2888.1 & 1.7 & 0.5 & 0.9541 \\
\hline $\mathrm{dwd} 3175$ & Q10-98 & 0.003 & 106 & 0.22 & 0.4 & 6531 & 0.5750 & 0.0014 & 16.956 & 0.045 & 2935.1 & 1.7 & 0.3 & 0.9143 \\
\hline $\mathrm{dwd} 3174$ & Q10-98 & 0.004 & 95 & 0.77 & 0.5 & 7074 & 0.5949 & 0.0016 & 18.621 & 0.055 & 3031.1 & 1.6 & 0.9 & 0.9449 \\
\hline dwd3169 & Q4-98 & 0.003 & 33 & 0.55 & 0.5 & 2254 & 0.6423 & 0.0022 & 22.856 & 0.081 & 3235.2 & 2.1 & 1.5 & 0.9298 \\
\hline
\end{tabular}

$\mathrm{Ab}$-abraded; zr-zircon grain; eq-equant; elong-elongate; clr-colourless; brn-brownish; crk-cracked; subrnd-subrounded; incl-inclusions, Pbcom is total measured common $\mathrm{Pb}$ assuming the isotopic composition of laboratory blank: 206/204-18.221; 207/204-15.612; 208/204-39.360 (errors of 2\%). Th/U calculated from radiogenic ${ }^{208} \mathrm{~Pb} /{ }^{206} \mathrm{~Pb}$ ratio and ${ }^{207} \mathrm{~Pb} /{ }^{206} \mathrm{~Pb}$ age assuming concordance. Disc. - per cent discordance for the given ${ }^{207} \mathrm{~Pb} /{ }^{206} \mathrm{~Pb}$ age. Uranium decay constants are from Jaffey et al. (1971).

$\mathrm{Lu} / \mathrm{Hf}$ value for the protolith. In the present case we calculate two 'end-member' mantle extraction ages based on the average $\mathrm{Lu} / \mathrm{Hf}$ ratios of crustal mafic rocks $(0.022$, see discussion in Amelin et al., 1999) and Precambrian granitoid rocks $(0.0093$, Vervoort and Patchett, 1996). Values of ${ }^{176} \mathrm{Lu} /{ }^{177} \mathrm{Hf}$ deter-

$\mathrm{Hf}$ isotopic data for detrital and igneous zircons

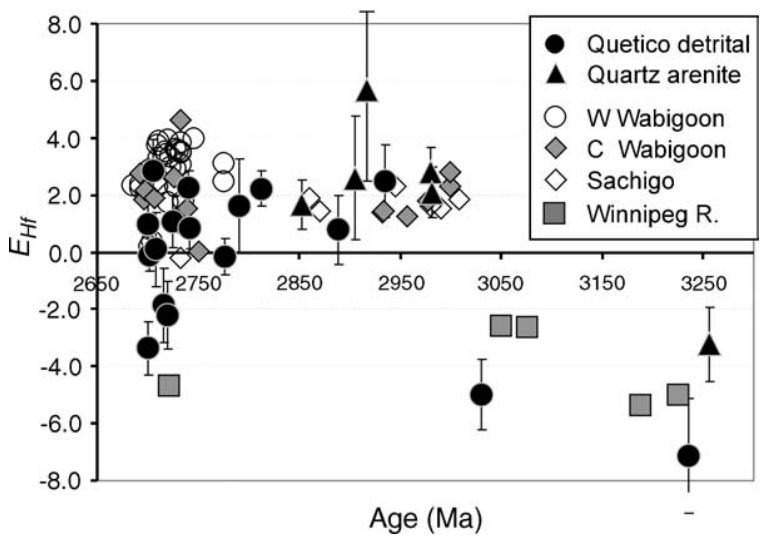

Fig. 5. Age vs. $\varepsilon_{\mathrm{Hf}}$ for middle and late Archean igneous rocks compared to detrital zircons from $>2.8 \mathrm{Ga}$ quartz arenites and $2.7 \mathrm{Ga}$ Quetico turbidities. mined from sedimentary rocks in the Superior province are $0.012-0.015$, which probably represents a good approximation to the Superior province crustal average and lies well within the range of these end member values (Stevensen and Patchett, 1990).

Model age calculations also depend on the parameters chosen to define the depleted mantle growth curve. Vervoort and Blichert-Toft (1999) suggested a model based on rocks with a wide range of ages whose $\varepsilon_{\mathrm{Hf}}$ values were calculated using the Siguigna et al. (1982) decay constant. The linear growth curve adopted for the present work is based on the ${ }^{176} \mathrm{Hf} /{ }^{177} \mathrm{Hf}$ ratio suggested by Vervoort and Blichert-Toft (1999) for present day depleted mantle $\left(\varepsilon_{\mathrm{Hf}}=18\right)$, and the mean $\varepsilon_{\mathrm{Hf}}$ value measured on rocks assumed to have formed from late Archean depleted mantle beneath the western Superior province ( $3.5 \pm 0.2$, see Section 5$)$. The first parameter falls near the upper (most depleted) limit of ${ }^{176} \mathrm{Hf} /{ }^{177} \mathrm{Hf}$ values for 'normal' mid-ocean ridge segments (Nowell et al., 1998). The second parameter is the best approximation for the mantle beneath the western Superior province and is therefore the most appropriate for defining mantle extraction ages in this region. This model implies a time integrated ${ }^{176} \mathrm{Lu} /{ }^{176} \mathrm{Hf}$ ratio of the MORB source of 0.0409 . Alternative models and 
implications for early growth of the continental crust are discussed below.

\subsection{Results from western Superior province terranes}

\subsubsection{Western Wabigoon subprovince}

The western Wabigoon subprovince contains the Kakagi Lake-Savant Lake volcanic belt (KSVB, Fig. 1). Values of $\varepsilon_{\mathrm{Hf}}$ for the central and southwestern part of KSVB show a high level of consistency (Fig. 3). All samples within the age range 2710-2732 Ma from this area are tightly grouped and give an average $\varepsilon_{\mathrm{Hf}}$ of $3.5 \pm 0.2$ (95\% confidence error, MSWD of 0.9 ). Late-tectonic plutons younger than $2710 \mathrm{Ma}$ in some cases appear to have slightly lower $\varepsilon_{\mathrm{Hf}}$ values and were omitted from this average. These plutons might be expected to show contamination from older crust because $2710 \mathrm{Ma}$ marks the beginning of regional deformation and deposition of orogenic sandstones, which commonly contain Mesoarchean detrital zircon. This supports previous suggestions that early volcanic rocks in this region mostly developed in a juvenile oceanic arc environment (Davis et al., 1988). Small amounts of enrichment appear in late plutons such as the Ottertail, Taylor Lake, Heronry and High Lake stocks.

Values of $\varepsilon_{\mathrm{Hf}}$ show less consistency for rocks in the northwestern part of the belt (Fig. 3). Zircon samples from a $2745 \mathrm{Ma}$ old felsic volcanic rock in the lower Handy Lake group and from a 2772 Ma old felsic volcanic rock in the Fourbay Lake Group have depleted Hf signatures ( +4 and +3 , respectively), which are similar to the arc-related volcanic rocks in the southwestern part of the belt. Evidence for slight but consistent enrichment is found from three 2732-2735 Ma rocks in this area. One is a subvolcanic quartz porphyry overlying the 2745 Ma volcanic unit within the Handy Lake Group (+1). The other two are a rhyolite $(+2)$ and a shallow pluton $(+1)$ associated with the South Sturgeon Lake volcanic assemblage. This assemblage is part of a caldera complex that may have formed in a back-arc environment and was host to significant volcanogenic massive sulphide mineralization at $2732 \mathrm{Ma}$ (Davis et al., 1985). A younger $2704 \mathrm{Ma}$ sub-volcanic porphyry near the top of the Handy Lake Group shows more enrichment and agrees with a similar-aged granitoid clast in a conglomerate along the nearby Kashaweogama Fault at +0 . This was probably eroded from a nearby pluton within the lower basaltic sequence of the Jutten Group. A quartz arenite unit within the upper part of the Jutten Group contains relatively old detrital zircons with ages ranging from $2.95 \mathrm{Ga}$ to $3.3 \mathrm{Ga}$. Hf analysis of one $3256 \mathrm{Ma}$ old detrital zircon from this unit gave an enriched $\varepsilon_{\mathrm{Hf}}$ value -3 , indicating possible mantle extraction ages in the range 3.4-3.5 Ga (Fig. 5). Such zircons are likely derived from rocks of the Winnipeg River subprovince (see Section 5).

\subsubsection{Central Wabigoon subprovince}

Tomlinson et al. (2001, 2003) suggested that the south-central part of the Wabigoon subprovince comprises a distinct terrane, which they called the Marmion terrane. This is based on evidence from zircon ages and $\mathrm{Nd}$ isotopes that the area is largely composed of juvenile and reworked 3.0 Ga crust (Davis and Jackson, 1988; Henry et al., 1998; Tomlinson et al., 2003). Evidence from $\mathrm{Hf}$ isotopes supports this view. Two of the oldest volcanic rocks from the Lumby Lake greenstone belt and a quartz diorite phase of the Marmion batholith give $3.0 \mathrm{Ga}$ ages and show the most primitive $\varepsilon_{\mathrm{Hf}}$ values (Fig. 4). These average to $2.7 \pm 0.4$. Seven younger plutons and a rhyolite in the age range 2.70-2.98 Ma show more enriched $\varepsilon_{\mathrm{Hf}}$ and give scattered values. Such a pattern would be expected for contamination from a crustal source that was not significantly older than $3.0 \mathrm{Ga}$.

Zircons from three Neoarchean felsic volcanic units in the Obonga Lake greenstone belt show variably depleted signatures for Hf (Figs. 3 and 4). This accords with $\mathrm{Nd}$ isotopic values for the corresponding whole rocks (Tomlinson et al., 2002). A 2739 Ma old foliated pluton intruded by the Roaring River complex (Stern et al., 1990) and quartz monzonite from the pluton itself show slightly enriched $\varepsilon_{\mathrm{Hf}}$ values of about +2 . Zircon from a $3075 \mathrm{Ma}$ old tonalite at Caribou Lake on the northern boundary of the Wabigoon subprovince shows a markedly enriched $\varepsilon_{\mathrm{Hf}}$ value of -2.6 , indicating a mantle extraction age in the range 3.3-3.4 Ga (Fig. 4). In contrast to the western Wabigoon subprovince, the central part contains rocks from different sources with a variety of crustal pre-histories.

\subsubsection{Winnipeg River subprovince}

Zircon $\varepsilon_{\mathrm{Hf}}$ data from rocks in the Winnipeg River subprovince show distinctly more enriched values than 
from most other samples in the western Superior province. One of the oldest units, the Cedar Lake gneiss, was dated at 3180 Ma by Corfu (1988). Subsequent $\mathrm{U}-\mathrm{Pb}$ dating of this unit by SHRIMP has shown that it contains a variety of zircons with ages extending back to $3250 \mathrm{Ma}$ (Melnyk et al., submitted). Two single zircons from the 3180 Ma sample dated by Corfu (1988) gave ${ }^{207} \mathrm{~Pb} /{ }^{206} \mathrm{~Pb}$ ages of $3188 \mathrm{Ma}$ and $3225 \mathrm{Ma}$. Both zircons have similar $\varepsilon_{\mathrm{Hf}}$ values of about -5 (Fig. 4). This indicates substantial enrichment, with a mantle extraction age in the range $3.5-3.7 \mathrm{Ga}$. A 3050 Ma old sample from the Tannis Lake gneiss also gave an enriched zircon $\varepsilon_{\mathrm{Hf}}$ value of -2.3 (Fig. 4) with a likely mantle extraction age in the range $3.2-3.4 \mathrm{Ga}$. These results are in broad agreement with $\mathrm{Nd}$ analyses from the same units, which gave mantle extraction ages of $3.4 \pm 0.2 \mathrm{Ga}$ (Henry et al., 2000). Ages of up to 3320 Ma have now been measured by U-Pb SHRIMP on zircons from a phase the Tannis Lake gneiss (Melnyk et al., submitted). Tonalite gneiss aged 2721 Ma near the southern boundary of the subprovince gave an enriched $\varepsilon_{\mathrm{Hf}}$ value of -5 (Fig. 3), suggesting a mantle extraction age for the crustal protolith in the range 3.1-3.3 Ga.

\subsubsection{Sachigo subprovince}

The most northerly sample in this study is a $2709 \mathrm{Ma}$ old shoshonitic rhyolite from the Oxford Lake Group in Manitoba. This gives a slightly enriched $\varepsilon_{\mathrm{Hf}}$ value of +2.5 (Fig. 3). Zircons from the North Caribou block in the Sachigo subprovince of Ontario show fairly uniform $\varepsilon_{\mathrm{Hf}}$ signatures of around +2 for different ages except for a Neoarchean tuff with $\varepsilon_{\mathrm{Hf}}$ of 0 (Fig. 4). Corfu and Stott (1993b, 1996) found juvenile Hf values for Mesoarchean rocks in the Uchi subprovince on the southern margin of the North Caribou block with a pattern of progressive scatter toward more enriched values in younger rocks. They suggested that these areas represent $3.0 \mathrm{Ga}$ crust that was progressively reworked with addition of considerable mantle-derived material at around $2.7 \mathrm{Ga}$. Given the small number of analyses, the present data may be consistent with this suggestion (Fig. 4).

Hf isotopic analyses were also carried out on single detrital zircons in quartz arenite units from the Sachigo subprovince. Five detrital zircons were analyzed from three units within the North Caribou block. Some of these analyses are relatively imprecise because of small sample size but they give $\varepsilon_{\mathrm{Hf}}$ values within error of the scatter of igneous rocks from the same area (Fig. 5). This supports previous suggestions based on age data that the quartz arenites had a local provenance.

\subsubsection{Quetico metasedimentary subprovince}

The Quetico subprovince represents a major E-W trending belt of metasedimentary and plutonic rocks that stretches across the western Superior province. About half the detrital zircon ages from this area are in the narrow age range of 2710-2700 Ma while others give scattered older ages, most of which are younger than 3.0 Ga. Quetico sandstones from the Rainy Lake area at the western end of the subprovince are an exception. They contain Meso- to Paleoarchean detrital zircons with ages as old as $3.45 \mathrm{Ga}$ (Fralick and Davis, 1999). Hf measurements on two detrital zircons with ages of $3031 \mathrm{Ma}$ and $3235 \mathrm{Ma}$ from this area gave enriched $\varepsilon_{\mathrm{Hf}}$ values of -4.7 and -6.3 , respectively (Fig. 5). The oldest indicates a mantle extraction age in the range 3.6-3.8 Ga. Both values are even more enriched than those found in rocks of similar age in the Winnipeg River subprovince. Neoarchean zircons show a spread from slightly enriched to an $\varepsilon_{\mathrm{Hf}}$ value of -3.0 (Fig. 5).

\section{Discussion}

\subsection{Terrane analysis and tectonic history}

Hf isotopic data from zircons confirm and expand on previous conclusions from $\mathrm{Nd}$ whole rock isotopic work (Henry et al., 1998, 2000), while adding the ability to reveal magmatic pre-histories of single detrital zircons. Based on these data, there appear to be at least three distinct terrane types in the western Superior province.

Juvenile 2.7-2.75 Ga terranes (e.g. western Wabigoon, Shebandowan subprovinces) probably formed largely in oceanic environments during the most intense phase of igneous activity in the region.

Juvenile $3.0 \mathrm{Ga}$ terranes (e.g. North Caribou, Marmion terranes) were reworked by continental arc and plume-related activity over a 300 m.y. time span, culminating in extensive granitoid magmatism at around $2.7 \mathrm{Ga}$. 
An older terrane, whose deep crustal level is exposed in the Winnipeg River subprovince, shows a protolith history that extends to $3.5 \mathrm{Ga}$. It was reworked during the Mesoarchean but seems to have largely escaped the most intense period of arc-related volcanism that occurred in most other areas in the age span 2.71-2.75 Ma. This terrane was again magmatically reworked during the main period of regional deformation that occurred in the time span $2.71-2.65 \mathrm{Ma}$ and affected the entire region.

Volcanic and plutonic rocks in the age range 2720-2730 Ma are present in most terranes. To test whether such rocks are autochthonous or whether they may have formed in an oceanic environment and been structurally juxtaposed against older units, $\varepsilon_{\mathrm{Hf}}$ values were compared for zircons from a number of terranes in this age range (Fig. 3). A single zircon Hf analysis from a $2722 \mathrm{Ma}$ volcanic unit in the Shebandowan greenstone belt, the western extension of the Wawa subprovince, shows $\varepsilon_{\mathrm{Hf}}$ of +4 . This is indistinguishable from the +3.5 value of many similar aged rocks in the western Wabigoon subprovince and is certainly indicative of minimal older crustal contamination. In contrast, the $2721 \mathrm{Ma}$ tonalite gneiss from the Winnipeg River subprovince shows $\varepsilon_{\mathrm{Hf}}$ of -4.9 , the most enriched value found from any Neoarchean rock in this study, with a 3.1-3.3 Ga range of mantle extraction ages that is comparable to $\mathrm{U}-\mathrm{Pb}$ zircon ages of the oldest gneisses in this terrane. Zircons from a $2723 \mathrm{Ma}$ old rhyolite in the Separation Lake greenstone belt give a less enriched $\varepsilon_{\mathrm{Hf}}$ value of -1 with mantle extraction ages in the range 2.9-3.1 Ga. This belt appears to be an eastern attenuated extension of the Bird River granitegreenstone subprovince and lies between the Winnipeg River and the English River subprovinces. The enriched Hf result could indicate that the Separation Lake belt volcanics are contaminated by much older Winnipeg River-type crust, which now lies to the south, or that they are correlative with rocks in the Uchi subprovince to the north. Finally, $2726 \mathrm{Ma}$ old zircon from a rhyolite in the Melchett Lake greenstone belt, an unusual exposure of volcanic rocks within the eastern English River subprovince, shows a primitive $\varepsilon_{\mathrm{Hf}}$ value of +3.8 . The relationship of the Melchett Lake belt to the younger $(<2704 \mathrm{Ma})$ surrounding metasedimentary rocks of the English River subprovince is unclear. If it represents exposed basement to these rocks then they were probably deposited onto oceanic or oceanic arc-related crust.
If it is allochthonous, then it may have been transported from terranes with similar age and provenance to the south. These data show that near contemporaneous magmatism was occurring in quite different tectonic environments over the time span 2720-2730 Ma. Evidence for autochthonous development of Neoarchean sequences in different Superior province terranes has been found in other U-Pb studies (Ayer et al., 2002).

Both the North Caribou block of the Sachigo subprovince and the Marmion terrane in the south-central part of the Wabigoon subprovince appear to consist largely of depleted mantle-derived $3.0 \mathrm{Ga}$ rocks and younger rocks derived from their reworking. Similarities in $\mathrm{Nd}$ and $\mathrm{Hf}$ isotopes, age distributions and lithological associations are close enough to warrant the suggestion that these two terranes are correlative. They could either represent rifted fragments of the same continent or the Marmion terrane might be a fragment of the North Caribou terrane that was transported southward during accretion of the Superior province. Re-assembly of near-contiguous rifted fragments during accretion seems less likely, given the destructive nature of plate collision processes and evidence for large-scale oblique convergence, which would offset once contiguous rifted fragments (Percival et al., 1994). The reflection seismic profile from line $1 \mathrm{~A}$ across the central Wabigoon subprovince suggests that the southcentral part contains a relatively thin upper crustal sheet overlying a more reflective middle crust (see southern part of section 1D in Fig. 2 of White et al., 2003). If so, the Marmion terrane may represent a klippe derived from the North Caribou terrane that was thrust over the accretionary margin. Emplacement of this terrane as a high-level thrust sheet at about $2700 \mathrm{Ma}$ could have provided a major source of sediment into the Quetico basin, which is consistent with Mesoarchean ages and moderately enriched $\mathrm{Hf}$ signatures from some of its detrital zircons.

The $\varepsilon_{\mathrm{Hf}}$ values of zircons in rocks from the Winnipeg River terrane generally show greater enrichment than those from other areas. Other distinctive features of the Winnipeg River subprovince are its relatively high metamorphic grade and plutonic character indicating that it was exhumed from depth, its much older $\mathrm{U}-\mathrm{Pb}$ ages, and the fact that it shows very few ages corresponding to the main 2.71-2.75 Ga period of calc-alkaline and tholeiitic magmatism that preceded accretion. Most rocks within the Winnipeg River sub- 
province are either younger than $2.71 \mathrm{Ga}$ or older than $2.8 \mathrm{Ga}$. This led Davis and Smith (1991) to suggest that the subprovince represents an accreted fragment of sialic crust that underthrust the Wabigoon subprovince. If so, it probably did not represent a continental terrane extensive enough to bring the accretionary process to an end. This is because the beginning of large scale melting and recumbant isoclinal folding (2716 Ma, Melnyk et al., submitted) predates what is thought to be the time of accretion of oceanic arcs in the Shebandowan subprovince further south $(<2700 \mathrm{Ma}$, Corfu and Stott, 1998). The seismic reflection profile also suggests that Winnipeg River crust was thrust southward over a slab of oceanic crust (White et al., 2003). The Winnipeg River subprovince may represent part of a small continental fragment that accreted early, as suggested in the model of Stott and Corfu (1991).

The enriched hafnium isotopic signature of a single 3256 Ma zircon from the Jutten quartz arenite (-3.2) in the northeastern part of KSVB suggests that its source was from rocks of the Winnipeg River subprovince. The Jutten sequence overlies 2884 Ma rhyolite (Skulski et al., 1999) and its youngest dated zircon gives $2948 \mathrm{Ma}$. It is likely that most such quartz arenites in the Superior province were deposited before $2800 \mathrm{Ma}$ since no detrital zircons younger than this have thus far been found in any of them despite the fact that younger rocks are voluminous in the region. Thus, Hf data support the suggestion of Skulski et al. (1999) that part of the Savant Lake area represents an early shelf sequence related to rocks of the Winnipeg River subprovince while rocks further south (2745 Ma lower Handy Lake and 2775 Ma Fourbay Lake units) were deposited in an oceanic environment. Hf in zircon from the $2.73 \mathrm{Ga}$ South Sturgeon Lake assemblage (Beidelman Bay pluton and felsic volcanic) shows slightly enriched signatures $(+1$ and +1.8 , respectively). $\mathrm{Nd}$ in whole rocks from the same area shows a similar level of enrichment (Bernier et al., 1999). These zircons agree in age and $\varepsilon_{\mathrm{Hf}}$ with a coeval part of the Savant Lake group $(+1.1)$, which suggests that the sequences are correlative. Since the South Sturgeon Lake assemblage is host to a number of major VMS deposits, this part of the Savant Lake group may be a useful exploration target. It is likely that these rocks evolved in a back-arc environment and their less depleted $\mathrm{Hf}$ and $\mathrm{Nd}$ may be due either to contamination with older crust (either Winnipeg River or Marmion-type terrane) or the influence of a plume source. The most enriched $\mathrm{Hf}$ values $\left(\varepsilon_{\mathrm{Hf}}\right.$ of about 0 ) are from zircons in $2.70 \mathrm{Ga}$ rocks. This period of magmatism is suggested to mark suturing between the older continental margin sequence and the oceanic rocks (Skulski et al., 1999).

Mesoarchean rocks are also found at Caribou Lake on the northern margin of the central Wabigoon subprovince (Davis et al., 1988) and in the OnamanTashota granite-greenstone belt east of Lake Nipigon (Stott and Davis, 1999). Zircon aged $3075 \mathrm{Ma}$ from Caribou Lake shows similar $\varepsilon_{\mathrm{Hf}}$ enrichment to the 3050 Ma zircon from gneiss at Tannis Lake near Kenora (Fig. 4). This supports the suggestion of Tomlinson et al. $(2001,2003)$ that rocks of the Winnipeg River terrane extend across much of the northern Wabigoon subprovince. The Jutten Group is at lower metamorphic grade than rocks of the Winnipeg River subprovince, which attain granulite facies at its eastern end near the Miniss River fault. This suggests that the Winnipeg River subprovince represents a deep crustal level of the Winnipeg River terrane that was differentially uplifted along the Miniss River fault. This uplift probably began as early as $2700 \mathrm{Ma}$, as suggested by detrital zircons from the western Quetico subprovince with Paleoarchean ages and enriched $\varepsilon_{\mathrm{Hf}}$ signatures.

Erosion of the Winnipeg River terrane at $2700 \mathrm{Ma}$ may account for the difference in the age distribution of detrital zircons from sandstones in the Quetico versus the English River subprovince. Turbidities in the English River subprovince were probably deposited in the time span 2700-2705 Ma based on ages of youngest detrital zircons and crosscutting plutons (Corfu et al., 1995; Davis, 1995), while lithologically similar rocks in the Quetico subprovince were deposited shortly after 2700 Ma (Davis, 1995; Davis et al., 1990). The age distribution of detrital zircons from the English River subprovince is similar to that of igneous rocks in the adjacent Wabigoon, Uchi and Sachigo subprovinces. It reflects the preponderance of igneous activity in these areas over the $2.71-2.75 \mathrm{Ga}$ time span with sparser but near continuous activity back to $3.0 \mathrm{Ga}$. In contrast, about half of the Quetico detrital zircon population is within the narrow age span 2700-2710 Ma. Plutonic rocks within this time span make up a significant proportion of the Winnipeg River subprovince and probably formed from extensive melting of older rocks. Uplift and exposure of these rocks to erosion at $2700 \mathrm{Ma}$ might have contributed much 
of the $2.70-2.71 \mathrm{Ga}$, isotopically enriched detrital zircon.

\subsection{Hf and $O$ isotopes in zircon}

Hf isotopic signatures of zircon from plutons younger than about $2710 \mathrm{Ma}$ scatter toward slightly more enriched values than older rocks from juvenile terranes. Many of these plutons are from the sanukitoid magmatic suite, which is thought to have formed from melting of mantle that had been metasomatized during previous subduction (Shirey and Hanson, 1984). The resulting magmas were modified by fractional crystallization and crustal assimilation (Stern et al., 1990; Stevenson et al., 1999). Emplacement of these plutons is usually approximately coeval with regional folding. The degree of Hf isotopic enrichment does not seem to be obviously correlated with the degree of fractionation or the crustal pre-history of the terranes in which the rocks were emplaced. King et al. (1998) noted that many late plutons show $\mathrm{O}$ isotopic ratios slightly higher than mantle values. They suggested that either crustal assimilation or dewatering of subducted sediments may have raised the $\mathrm{O}$ isotopic signature of the metasomatized mantle source from which the primary magmas of the plutons were derived. Hf isotopes provide a potential method for evaluating these possibilities. Fig. 6 shows $\delta^{18} \mathrm{O}$ plotted against $\varepsilon_{\mathrm{Hf}}$ for zircons analysed by King et al. (1998). This includes data from some

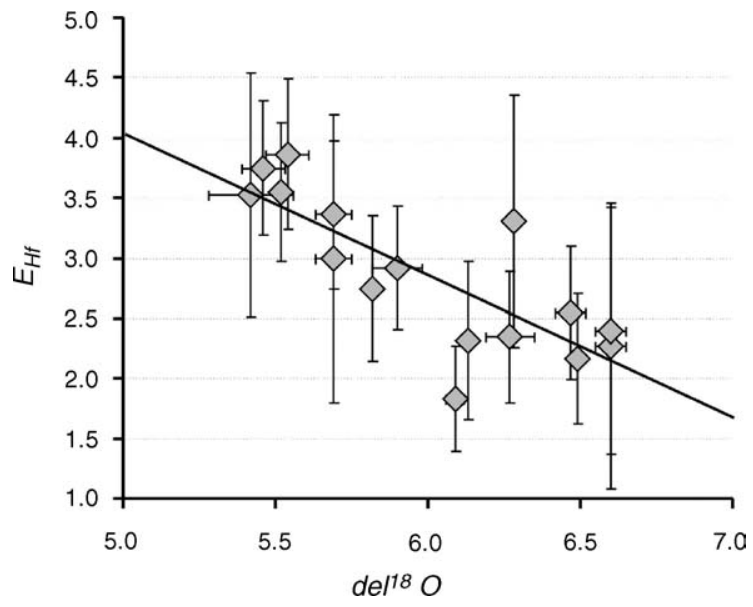

Fig. 6. $\varepsilon_{\mathrm{Hf}}$ vs. $\delta^{18} \mathrm{O}$ for zircons from plutons in the western Superior Transect area along with the best fit correlation line. Data with $\delta^{18} \mathrm{O}$ $>5.5$ are mostly from ca. $2.70 \mathrm{Ga}$ rocks of the sanukitoid suite. pre-2.71 Ga TTG rocks, which show mantle-like $\delta^{18} \mathrm{O}$ values around $5.5 \%$ and depleted $\varepsilon_{\mathrm{Hf}}$ values. Rocks with both elevated $\delta^{18} \mathrm{O}$ values and slightly enriched $\varepsilon_{\mathrm{Hf}}$ are largely from the late-tectonic sanukitoid suite. Although the relatively large errors in $\varepsilon_{\mathrm{Hf}}$ make it difficult to establish a well-constrained trend (correlation coefficient of 0.79), there appears to be an inverse correlation between $\delta^{18} \mathrm{O}$ and $\varepsilon_{\mathrm{Hf}}$. The best-fit line follows the equation $\varepsilon_{\mathrm{Hf}}=-1.2 \times \delta^{18} \mathrm{O}+9.9$. This correlation suggests that the increase in $\delta^{18} \mathrm{O}$ was more likely due crustal assimilation rather than dewatering of sediments because $\mathrm{Hf}$ resides mostly in zircon and should be immobile during hydrothermal processes. Stevenson et al. (1999) also suggested on geochemi$\mathrm{cal}, \mathrm{Nd}$ and $\mathrm{Pb}$ isotope grounds that crustal assimilation played a major role in the magmatic evolution of the sanukitoid suite. If the most differentiated plutons (quartz monzonite) represent 50\% contamination, this would imply a contaminant with $\delta^{18} \mathrm{O}$ of about $9 \%$ and a $\varepsilon_{\mathrm{Hf}}$ value of about 0 . This is consistent with $\delta^{18} \mathrm{O}$ values measured on orogenic sandstones in metasedimentary subprovinces such as the Pontiac (Feng et al., 1990) as well as Hf isotopic measurements on bulk zircon from these rocks (Stevensen and Patchett, 1990). It would also be consistent with lesser amount of contamination from more $\delta^{18} \mathrm{O}$ enriched intravolcanic metasedimentary units (Feng et al., 1990) with an older mantle extraction age. Sanukitoid plutons are often spatially associated with such units.

\subsection{Implications for development of the Neoarchean depleted mantle: choice of the ${ }^{176} \mathrm{Lu}$ decay constant}

Fourteen Neoarchean units in the western Wabigoon subprovince that show no evidence of crustal contamination define a consistent $\varepsilon_{\mathrm{Hf}}$ value of $3.5 \pm 0.2$ (MSWD of 0.86 , average age of $2724 \mathrm{Ma}$ ). This likely represents the sub-oceanic depleted mantle at this time. Omitted from the western Wabigoon data set are five relatively young plutons that may have been affected by older crust (see previous section) and one $2720 \mathrm{Ma}$ old rhyolite from the Lake of the Woods area that appears somewhat enriched. Measured $\varepsilon_{\mathrm{Hf}}$ from a $2.72 \mathrm{Ga}$ volcanic unit in the Shebandowan belt is consistent with this value, as is the average $\varepsilon_{\mathrm{Hf}}$ value of $4.0 \pm 0.4$, adjusted for the presently assumed constants, that was measured for units in the southern Abitibi subprovince 
by Corfu and Noble (1992). These areas are now about $1000 \mathrm{~km}$ apart but considering that they probably formed in an oceanic environment before accretion, they may have originally been much more widely separated. This suggests that the Neoarchean depleted mantle source was quite uniform with respect to its $\mathrm{Hf}$ isotopic composition.

The absolute value of the Hf isotopic anomaly depends strongly on the choice of ${ }^{176} \mathrm{Lu}$ decay constant. Until recently, a value of $1.931 \pm 0.027 \times 10^{-5}$ m.y. ${ }^{-1}$, based on $\gamma-\gamma$ coincidence counting (Siguigna et al., 1982) was widely used. However, radioactive counting experiments over the past two decades have not produced consistent values (Scherer et al., 2003). The analytical precision and accuracy of the ${ }^{238} \mathrm{U}$ and ${ }^{235} \mathrm{U}$ decay constants $( \pm 0.2 \%$, Jaffey et al., 1971 ; Mattinson, 1987) are much better established than that of ${ }^{176} \mathrm{Lu}$, making comparative U-Pb and $\mathrm{Lu}-\mathrm{Hf}$ dating an attractive calibration method. The value of $1.865 \pm 0.015 \times 10^{-5} \mathrm{~m} . \mathrm{y} .^{-1}$ used in this work is based on $\mathrm{U}-\mathrm{Pb}$ and $\mathrm{Lu}-\mathrm{Hf}$ dating of the same mineral phases in terrestrial rocks (Scherer et al., 2001). More recently, Bizzarro et al. (2003) have proposed a higher value of $1.983 \pm 0.033 \times 10^{-5}$ m.y. ${ }^{-1}$ based on comparative $\mathrm{U}-\mathrm{Pb}$ and $\mathrm{Lu}-\mathrm{Hf}$ dating of meteorites. The ca. $6 \%$ difference between terrestrial and meteoritic samples has been reproduced by other measurements on similar samples (Scherer et al., 2003; Söderlund et al., 2003; Blichert-Toft et al., 2002), implying that there is some hidden bias with either the terrestrial or meteoritic samples. The effects of using different proposed decay constants on the calculated chondritic growth curve for $\mathrm{Hf}$ become progressively greater with age and result in major differences for calculated $\varepsilon_{\mathrm{Hf}}$ values from Archean samples. For example, The Scherer et al. (2001) value produces $\varepsilon_{\mathrm{Hf}}$ values that are 2.2 units lower at $2700 \mathrm{Ma}$ than the Siguigna et al. (1982) value and 3.9 units lower than that of Bizzarro et al. (2003). We follow Scherer et al. (2001) in this work because it is presently the best-replicated value. It is in tight agreement with several other determinations on a variety of different aged minerals and whole rocks including rocks comparable in age to those of the present study (Scherer et al., 2003; Söderlund et al., 2003). The consistency of this choice with other data and observations is discussed below.

Fig. 7 shows estimates of $\varepsilon_{\mathrm{Hf}}$ for depleted mantle at $2.7 \mathrm{Ga}$ and $3.0 \mathrm{Ga}$ based on the current Superior

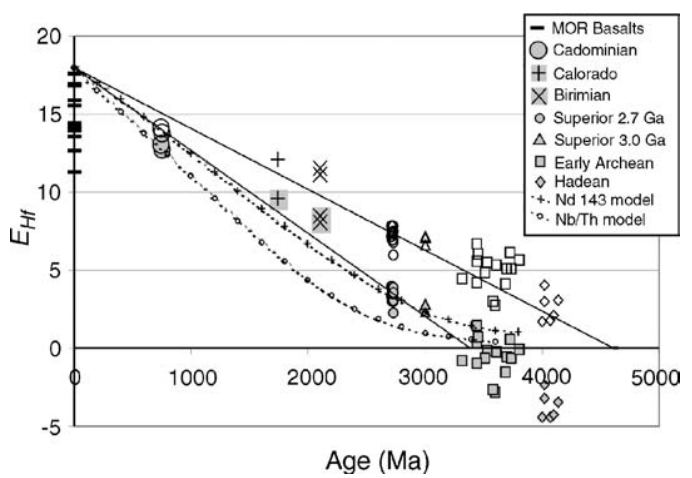

Fig. 7. Comparison of data calculated assuming a ${ }^{176} \mathrm{Lu}$ decay constant of $1.865 \times 10^{-5}$ m.y. ${ }^{-1}$ (lower data set, Scherer et al., 2001 ), and a decay constant of $1.983 \times 10^{-5} \mathrm{~m} . \mathrm{y}^{-1}$ (upper data set, Bizzarro et al., 2003). Linear depleted mantle growth models are shown for each proposed value assuming a present day ${ }^{176} \mathrm{Hf} /{ }^{177} \mathrm{Hf}$ ratio of $0.28325\left(\varepsilon_{\mathrm{Hf}}=+18\right)$, based on the upper limit of MORB basalts, and ${ }^{176} \mathrm{Hf} /{ }^{177} \mathrm{Hf}$ measured for $2.7 \mathrm{Ga}$ zircons in mantle-derived rocks from this study. Data from mantle-derived samples in other areas are shown for comparison (MOR-Nowell et al., 1998; Cadominian-Samson et al., 2003; Colorado-Vervoort and Blichert-Toft, 1999; Birimian-Blichert-Toft et al., 1999; early Archean-Amelin et al. (2001); Vervoort and Blichert-Toft, 1999; Hadean-Amelin et al., 1999). Also shown are depleted mantle evolution models based on ${ }^{143} \mathrm{Nd}$ isotopes (Nagler and Kramers, 1998) and time integrated $\mathrm{Nb} / \mathrm{Th}$ ratios (Collerson and Kamber, 1999), both curves adjusted to have the same present day value as $\varepsilon_{\mathrm{Hf}}$.

province data, as well as a previously published reference data set from zircon that ranges in age over most of the geologic time scale. These data are shown calculated using both the Scherer et al. (2001) and the Bizzarro et al. (2003) decay constants. The reference data are not comprehensive but were chosen for comparison purposes from previous studies and compilations (Amelin et al., 1999, 2000; Vervoort and Blichert-Toft, 1999) as generally representing the most depleted results on zircon. Scattered data from some of the early Archean rocks and Hadean detrital zircons may include effects of recycling.

The Superior province data for depleted mantle are approximately consistent with the roughly linear distribution of post-Archean data no matter which decay constant is used (Fig. 7) but the choice of decay constant greatly affects the intersection age with the chondritic line (horizontal axis). Using the Scherer et al. (2001) decay constant, a linear depleted mantle growth model based on $\varepsilon_{\mathrm{Hf}}$ of +3.5 at $2.7 \mathrm{Ga}$ and a depleted mantle estimate of +18 at $0 \mathrm{Ma}$ (Vervoort and 
Blichert-Toft, 1999) intersects the chondritic evolution line at an age of $3380 \mathrm{Ma}$. Linear models such as this assume that formation of the continental crust and associated mantle depletion occurred during one event whereas it is likely to have occurred progressively but with the bulk of crust-mantle extraction completed by the end of the Archean (Condie, 1997). The mean $\varepsilon_{\mathrm{Hf}}$ value of $+2.7 \pm 0.4 \mathrm{Ma}$ measured from three $3.0 \mathrm{Ga}$ old units in the south-central Wabigoon subprovince falls above the extrapolated line suggesting a lower degree of mantle depletion at this time and an earlier age for the beginning of mantle differentiation. A growth line between Superior province $\varepsilon_{\mathrm{Hf}}$ values at $3000 \mathrm{Ma}$ and $2700 \mathrm{Ma}$ would intersect the chondritic line at 3920 Ma. Despite their simplistic assumptions, these models show that the Scherer et al. (2001) decay constant value produces results that are broadly consistent with the preserved early rock record. Rocks older than $3.5 \mathrm{Ga}$ are extremely rare and the oldest rock yet dated is about $4.0 \mathrm{Ga}$ (Bowring and Williams, 1999; Stern and Bleeker, 1998). In contrast, data based on the Bizzarro et al. (2003) decay constant value imply sizeable amounts of mantle depletion during the early Archean and Hadean. A one stage linear model based on this decay constant gives a chondritic intersection age of $4.6 \mathrm{Ga}$ (Fig. 7).

Comparison of mantle evolution curves for $\mathrm{Nd}$ and $\mathrm{Hf}$ isotopes provides one independent way to test for a realistic choice of decay constant. The ${ }^{147} \mathrm{Sm}$ decay constant is considered to be accurate and both parent-daughter pairs generally show a strong geochemical coherence so the isotopic systems should produce mantle evolution curves of the same shape. The average ${ }^{143} \mathrm{Nd}$ mantle evolution curve suggested by Nagler and Kramers (1998) is compared to $\varepsilon_{\mathrm{Hf}}$ data on Fig. 7 where its vertical axis (not numbered) has been scaled so $\varepsilon_{\mathrm{Hf}}$ and $\varepsilon_{\mathrm{Nd}}$ match at $0 \mathrm{Ma}$. The depleted mantle $\varepsilon_{\mathrm{Hf}}$ data show good agreement with the $\mathrm{Nd}$ curve for Mesoarchean and younger rocks when they are calculated using the Scherer et al. (2001) decay constant value but poor agreement when calculated using the Bizzarro et al. (2003) value. Thus, despite evidence for the existence of Paleoarchean and Hadean mantle depletion (Boyet et al., 2003; Caro et al., 2003; Bennett et al., 1993; Jacobsen and Dymek, 1988), the depleted mantle Nd isotopic record from middle Archean time to the present supports the Scherer et al. (2001) decay constant value.
Another comparative approach can be made using trace element ratios such as $\mathrm{Nb} / \mathrm{Th}$ that are sensitive to crust-mantle differentiation. Because Th is preferentially enriched in the continental crust, the depleted mantle residue develops a supra-chondritic $\mathrm{Nb} / \mathrm{Th}$ ratio, which has been used as a proxy for the degree of mantle depletion (Collerson and Kamber, 1999; Kamber et al., 2003a). For a two-component system (enriched crust and depleted mantle), the integral of the $\mathrm{Nb} / \mathrm{Th}$ secular variation curve for rocks derived from depleted mantle should be similar to the timeintegrated history of mantle depletion as recorded by the growth of ${ }^{176} \mathrm{Hf}$ and ${ }^{143} \mathrm{Nd}$ isotopic anomalies. The integral curve presented in Fig. 7 is based on $\mathrm{Nb} / \mathrm{Th}$ ratios compiled by Collerson and Kamber (1999) minus the bulk silicate Earth ratio of 6.96 suggested by Kamber et al. (2003b). As with $\mathrm{Nd}$, the curve is scaled to give the same value as the $\varepsilon_{\mathrm{Hf}}$ curve at $0 \mathrm{Ma}$. This curve appears somewhat inconsistent with data calculated using either the Scherer et al. (2001) or Bizzarro et al. (2003) ${ }^{176} \mathrm{Lu}$ decay constant values, although it is closer to those using the Scherer et al. (2001) value. Agreement would be improved if the bulk earth value of $\mathrm{Nb} / \mathrm{Th}$ were somewhat lower than that estimated by Kamber et al. (2003b). Long-term storage of $\mathrm{Nb}$ in a high $\mathrm{Nb} / \mathrm{Th}$ reservoir such as subducted ocean crust could also explain the difference. This would tend to offset the effect of transferring Th to the continental crust and delay substantial $\mathrm{Nb} / \mathrm{Th}$ fractionation in the Neoarchean and Paleoproterozoic mantle. In this case $\mathrm{Nb} / \mathrm{Th}$ evolution could have been largely controlled by re-mixing of subducted ocean crust into the depleted mantle reservoir by convection or plumes. This mechanism was discussed by Kamber et al. (2003b), although they concluded that it had little effect on the continental growth curve.

Hf data from Hadean zircons analyzed by Amelin et al. (1999) show negative $\varepsilon_{\mathrm{Hf}}$ values when calculated using the Scherer et al. (2001) decay value (Fig. 7). This implies that at least some of the zircons had significantly older protoliths, which must have been felsic in order for their Hf model ages to be less than the age of the Earth. This is consistent with observations that some $>4.0 \mathrm{Ga}$ zircons contain $\mathrm{K}$-feldspar and quartz inclusions, significantly older cores, and high $\delta^{18} \mathrm{O}$ ratios (Maas et al., 1992; Wilde et al., 2001; Mojzsis et al., 2001). The Bizzarro et al. (2003) value would give depleted mantle signatures for these zircons. ${ }^{143} \mathrm{Nd}$ and 
${ }^{142} \mathrm{Nd}$ isotopes in rocks from Isua (Boyet et al., 2003; Caro et al., 2003) as well as $\mathrm{Pb}$ isotopic data in galena (Appel et al., 1978; Frei and Rosing, 2001; Kamber et al., 2003b) suggest that depleted mantle existed during the Hadean and that it had a highly enriched crustal compliment. By the end of the Hadean, such early crust must have been mostly recycled into the mantle, erasing its effect on Neoarchean and younger rocks (Kamber et al., 2003b). Formation of a depleted mantle with high Lu/Hf occurred on the Moon following solidification of its magma ocean, as shown by Archean-aged basalts with $\mathrm{Hf}$ isotopes that are much more radiogenic than in coeval terrestrial rocks (Unruh et al., 1984; Beard et al., 1998). A similar mantle residue produced from solidification of a terrestrial magma ocean would have rapidly produced high $\varepsilon_{\mathrm{Hf}}$ and $\varepsilon_{\mathrm{Nd}}$ values before foundering of the early crust. If the Scherer et al. (2001) decay constant value is correct, there remains no memory of this period of early differentiation in mantle beneath the Superior province. Hadean crust appears to have been recycled into the mantle with great efficiency resulting in a uniform isotopic composition that persisted to the Neoarchean.

\section{Conclusions}

The southwest part of the Wabigoon subprovince is largely composed of juvenile $2.7 \mathrm{Ga}$ crust. The southern part of the central Wabigoon may be correlative with rocks of the North Caribou terrane in the Sachigo subprovince. Both consist of juvenile $3.0 \mathrm{Ga}$ crust that was subsequently reworked by arc and plume magmatism. The Winnipeg River subprovince appears unique in the western Superior province in containing an isotopic record of early Archean crust that extends back to at least $3.5 \mathrm{Ga}$. Younger rocks from the Winnipeg River subprovince contain a significant component of recycled ancient crust. The northern Wabigoon subprovince also contains rocks with middle to early Archean magmatic provenance and, along with evidence from Nd isotopes, this may indicate that the Winnipeg River terrane extends across the northern part of the Wabigoon subprovince. Juvenile rocks show reproducible depleted mantle $\varepsilon_{\mathrm{Hf}}$ values indicating that the Hf method on zircon, like $\mathrm{U}-\mathrm{Pb}$ dating, is robust. The ability to determine initial $\mathrm{Hf}$ isotopic compositions on single detrital zircons is a unique advantage of the method and shows that some of the early quartz arenite sequences and late orogenic sediments were derived from recycled crust with a history extending to the early Archean.

Results of multi-collector ICP-MS Hf isotopic analyses on zircon are broadly consistent with previous $\mathrm{Nd}$ isotopic studies and TIMS-based Hf studies in the Superior province. They support previous suggestions that the western Superior region was assembled by a process that involved recycling of older crust in the north and accretion of juvenile oceanic terranes, and possibly Paleoarchean sialic fragments, from the south.

Uncertainty in the ${ }^{176} \mathrm{Lu}$ decay constant is a major problem for using $\mathrm{Hf}$ isotopic data to study early crust-mantle differentiation. Calculating data from mantle-derived rocks using the ${ }^{176} \mathrm{Lu}$ decay constant of Scherer et al., (2001) gives $\varepsilon_{\mathrm{Hf}}$ compositions for the depleted mantle reservoir of $3.5 \pm 0.2$ at $2.72 \mathrm{Ga}$ and $2.7 \pm 0.4$ at $3.0 \mathrm{Ga}$. Linear models for mantle depletion using these parameters suggest that significant continental crust formation began in the early Archean, not the Hadean. The higher Bizzarro et al. (2003) decay constant indicates significant mantle depletion early in the Hadean, consistent with ${ }^{143} \mathrm{Nd}$ and ${ }^{143} \mathrm{Nd}$ isotopic evidence from the oldest rocks, but less consistent with post-Archean $\mathrm{Nd}$ mantle evolution than the Scherer et al. (2001) value. The Bizzarro et al. (2003) value implies long-term persistence of Hadean mantle depletion, whereas the Scherer et al. (2001) value can only be consistent with Hadean mantle depletion if the earliest enriched crust became remixed with its mantle source early in the Archean, leaving little or no evidence of its existence in younger rocks. Both cases support suggestions by Armstrong (1991) that large amounts of enriched crust existed near the beginning of Earth's history. Continuing Hf isotopic work on precisely dated zircon will be a powerful tool for resolving regional and global problems in Earth history, but establishing a precise and unambiguous value for the ${ }^{176} \mathrm{Lu}$ decay constant is essential to its effective use in studying early crust-mantle differentiation.

\section{Acknowledgements}

U-Pb chemistry was carried out by Galina Amelina. Matt Horstwood is thanked for assistance in maintaining the Plasma 54. The manuscript was greatly 
improved by comments from J. Blichert-Toft, B. Kamber and V. Bennett. Financial assistance from the Western Superior Lithoprobe Transect project is gratefully acknowledged. This is Lithoprobe publication number 1431.

\section{References}

Amelin, Y., Lee, D.-C., Halliday, A.N., Pidgeon, R.T., 1999. Nature of the Earth's earliest crust from hafnium isotopes in single zircons. Nature 399, 252-255.

Amelin, Y., Lee, D.-C., Halliday, A.N., 2000. Early-middle Archean crustal evolution deduced from $\mathrm{Lu}-\mathrm{Hf}$ and $\mathrm{U}-\mathrm{Pb}$ isotopic studies of single zircon grains. Geochim. Cosmochim. Acta 64, 4205-4225.

Appel, P.W.U., Moorbath, S., Taylor, P.N., 1978. Least radiogenic terrestrial lead from Isua, West Greenland. Nature 272, 524526.

Armstrong, R.L., 1991. The persistent myth of crustal growth. Aust. J. Earth Sci. 38, 613-630.

Ayer, J.A., Davis, D.W., 1997. Sequential evolution of an Archean intra-oceanic arc, an sland arc and a shoshonitic arc: geochronological and geochemical evidence from the Lake of the Woods greenstone belt, northwest Ontario. Precambrian Res. 81, 155-178.

Ayer, J., Amelin, Y., Corfu, F., Kamo, S., Ketchum, J., Kwok, K., Trowell, N., 2002. Evolution of the Abitibi greenstone belt: early interaction between plume- and subduction-related volcanism followed by sedimentation, folding and plutonism associated with accretion to the Superior Province craton. Precambrian Res. $115,63-95$.

Beard, B.L., Taylor, L.A., Scherer, E.E., Johnson, C.M., Snyder, G.A., 1998. The source region and melting mineralogy of high titanium and low-titanium lunar basalts deduced from $\mathrm{Lu}-\mathrm{Hf}$ isotopic data. Geochim. Cosmochim. Acta. 62, 525-544.

Bennett, V.C., Nutman, A.P., McCulloch, M.T., 1993. Nd isotopic evidence for transient highly depleted mantle reservoirs in the early history of the Earth. Earth Planet. Sci. Lett. 119, 299-317.

Bernier, F., Stevensen, R.K., Gariepy, C., Franklin, J.M., 1999. $\mathrm{Nd}$ isotopic studies in the south Sturgeon Lake greenstone belt: a progress report. In: Harrap, R.M., Helmstaedt, H.H. (Eds.), Western Superior Transect Fifth Annual Workshop. Lithoprobe Report 70, Lithoprobe Secretariat, University of British Columbia, pp. 117-121.

Bizzarro, M., Baker, J.A., Haack, J., Ulfbeck, D., Rosing, M., 2003. Early history of Earth's crust-mantle system inferred from hafnium isotopes in chondrites. Nature 421, 931-933.

Blichert-Toft, J., Albarède, F., 1997. The Lu-Hf isotope geochemistry of chondrites and the evolution of the crust-mantle system. Earth Planet. Sci. Lett. 148, 243-258.

Blichert-Toft, J., Albarède, F., Rosing, M., Frei, R., Bridgewater, D., 1999. The $\mathrm{Nd}$ and $\mathrm{Hf}$ isotopic evolution of the mantle through the Archean. Results from the Isua supracrustals, West Greenland, and from the Birimian terranes of West Africa. Geochim. Cosmochim. Acta 63, 3901-3914.
Blichert-Toft, J., Boyet, M., Télouk, P., Albarède, F., 2002. 147Sm$143 \mathrm{Nd}$ and $176 \mathrm{Lu}-176 \mathrm{Hf}$ in eucrites and the differentiation of the HED parent body. Earth Planet. Sci. Lett. 204, 167181.

Bowring, S.A., Williams, I.S., 1999. Priscoan (4.00-4.03 Ga) orthogneisses from northwestern Canada. Contrib. Mineral. Petrol. 134, 3-16.

Boyet, M., Blichert-Toft, J., Rosing, M., Storey, M., Telouk, P., Albarede, F., 2003. $142^{\mathrm{Nd}}$ evidence for early Earth differentiation. Earth Planet. Sci. Lett. 214, 427-442.

Card, K.D., 1990. A review of the Superior province of the Canadian shield, a product of Archean accretion. Precambrian Res. 48, 99-156.

Card, K.D., Ciesielski, A., 1986. DNAG \#1 Subdivisions of the Superior province of the Canadian shield. Geosci. Canada 13, 5-13.

Caro, G., Bourdon, B., Birck, J.-L., Moorbath, S., 2003. ${ }^{146} \mathrm{Sm}_{-}{ }^{142} \mathrm{Nd}$ evidence from Isua metamorphosed sediments for early differentiation of the Earth's mantle. Nature 423, 428-432.

Collerson, K.D., Kamber, B.S., 1999. Evolution of the continents and the atmosphere inferred from Th-U-Nb systematics of the depleted mantle. Science 283, 1519-1522.

Condie, K.C., 1997. Plate Tectonics and Crustal Evolution, fourth ed. Butterworth-Heineman, $282 \mathrm{pp}$.

Corfu, F., 1988. Differential response of U-Pb systems in coexisting accessory minerals, Winnipeg River Subprovince, Canadian Shield: implication for Archean crustal growth and stabilization. Contrib. Mineral. Petrol. 98, 312-325.

Corfu, F., Noble, S.R., 1992. Genesis of the southern Abitibi greenstone belt, Superior province, Canada: evidence from zircon $\mathrm{Hf}$ isotopic analyses using a single filament technique. Geochim. Cosmochim. Acta 56, 2081-2097.

Corfu, F., Stott, G.M., 1996. Hf isotopic composition and age constraints on the evolution of the Archean central Uchi subprovince, Ontario, Canada. Precambrian Res. 78, 53-63.

Corfu, F., Stott, G.M., 1993a. Age and petrogenesis of two late Archean magmatic suites, northwestern Superior province, Canada: Zircon U-Pb and Lu-Hf isotopic relations. J. Petrol. 34, 817-838.

Corfu, F., Stott, G.M., 1998. The Shebandowan greenstone belt, western Superior Province: U-Pb ages, tectonic implications and correlations. Geol. Soc. Am. Bull. 110, 1467-1484.

Corfu, F., Stott, G.M., 1993b. U-Pb geochronology of the central Uchi subprovince, Superior Province. Can. J. Earth Sci. 30, 1179-1196.

Corfu, F., Stott, G.M., Breaks, F.W., 1995. U-Pb geochronology and evolution of the English River Subprovince, an Archean low Phigh T metasedimentary belt of the Superior Province. Tectonics 14, 1226-1233.

Cruden, A.R., Davis, D.W., Menard, T., Robin, P.-Y.F., 1997. Structural and geochronological relationships between the Winnipeg River and Wabigoon subprovinces: implications for the terrane accretion model. In: Harrap, R.M., Helmstaedt, H.H. (Eds.), Western Superior Transect Third Annual Workshop, Lithoprobe Report 63, Lithoprobe Secretariat, University of British Columbia, pp. 18-26.

Davis, D.W., 2002. U-Pb geochronology of Archean metasediments in the Pontiac and Abitibi subprovinces, Quebec, constraints on 
timing, provenance and regional tectonics. Precambrian Res. 115, 97-117.

Davis, D.W., 1999. Report on U-Pb geochronology of rocks from the Onaman Lake belt. Unpublished report to the Ontario Geological Survey, March 30, 1999. 6 p., 2 tab., 12 fig.

Davis, D.W., 1998. Speculations on the formation and crustal structure of the Superior province from $\mathrm{U}-\mathrm{Pb}$ geochronology. In: Harrap, R.M., Helmstaedt, H.H. (Eds.), Western Superior Transect Fourth Annual Workshop. Lithoprobe Report 65, 21-28, Lithoprobe Secretariat, University of British Columbia.

Davis, D.W., 1995. Provenance and depositional age constraints on sedimentation in the western Superior transect area from $\mathrm{U}-\mathrm{Pb}$ ages of zircons. In: Harrap, R.M., Helmstaedt, H.H. (Eds.), Western Superior Transect Second Annual Workshop. Lithoprobe Report 53, 18-23, Lithoprobe Secretariat, University of British Columbia.

Davis, D.W., Edwards, G.R., 1986. Crustal evolution of Archean rocks in the Kakagi Lake area, Wabigoon Subprovince, Ontario, as interpreted from high-precision U-Pb geochronology. Can. J. Earth Sci. 23, 182-192.

Davis, D.W., Edwards, G.R., 1985. Grant 179-The petrogenesis and metallogenesis of the Atikwa-Lawrence volcanic-plutonic terrain. Ontario Geoscience Research Program Summary of Research, Ontario. Geol. Surv. Misc. Paper 127, 101-111.

Davis, D.W., Edwards, G.R., 1982. Zircon U-Pb ages from the Kakagi Lake area, Wabigoon Subprovince, northwest Ontario. Can. J. Earth Sci. 19, 2135-2145.

Davis, D.W., Jackson, M.C., 1988. Geochronology of the Lumby Lake greenstone belt: a $3 \mathrm{Ga}$ complex within the Wabigoon Subprovince, northwest Ontario. Geol. Soc. Am. Bull. 100, 818-824.

Davis, D.W., Moore, M., 1991. Geochronology in the western Superior Province. Royal Ontario Museum unpublished report for Ontario Geological Survey.

Davis, D.W., Smith, P.M., 1991. Archean gold mineralization in the Wabigoon subprovince, a product of crustal accretion: evidence from precise U-Pb geochronology in the Lake of the Woods area, Superior Province, Canada. J. Geol. 99, 337-353.

Davis, D.W., Stott, G.M., 2002. Project unit 89-47. Geochronology of several greenstone belts in the Sachigo subprovince, northwestern Ontario. Ontario Geological Survey Summary of Field Work 2001, 13 pp.

Davis, D.W., Trowell, N.F., 1982. U-Pb ages from the eastern Savant Lake-Crow Lake metavolcanic-metasedimentary belt, northwest Ontario. Can. J. Earth Sci. 19, 868-877.

Davis, D.W., Krogh, T.E., Hinzer, J., Nakamura, E., 1985. Zircon dating of polycyclic volcanism at Sturgeon Lake and implications for base metal mineralization. Econ. Geol. 80, 1942-1952.

Davis, D.W., Pezzutto, F., Ojakangas, R.W., 1990. The age and provenance of metasedimentary rocks in the Quetico Subprovince, Ontario, from single zircon analyses: implications for Archean sedimentation and tectonics in the Superior Province. Earth Planet. Sci. Lett. 99, 195-205.

Davis, D.W., Poulsen, K.H., Kamo, S.L., 1989. New insights into Archean crustal development from geochronology in the Rainy Lake area, Superior Province, Canada. J. Geol. 97, 379-398.

Davis, D.W., Sutcliffe, R.H., Trowell, N.F., 1988. Geochronological constraints on the tectonic evolution of a late Archean greenstone belt, Wabigoon subprovince, northwest Ontario. Precambrian Res. 39, 171-191.

Dickin, A.P., McNutt, R.H., Mueller, H., Beakhouse, G.P., 1990. Evidence for an early Archean cratonic nucleus in the Superior Province of Ontario. Seventh international conference on Geochronology, cosmochronology and isotope geology. Abstracts-Geol. Soc. Aust. 27, 27.

Feng, R., Kerrich, R., Maas, R., 1990. Geochemical, oxygen, and neodymium isotopic compositions of metasediments from the Abitibi greenstone belt and Pontiac subprovince, Canada: evidence for ancient crust and Archean terrane juxtaposition. Geochim. Cosmochim. Acta 57, 641-658.

Fralick, P., Davis, D., 1999. The Seine-Coutchiching problem revisited: sedimentology, geochronology and geochemistry of sedimentary units in the Rainy Lake and Sioux Lookout areas. In: Harrap, R.M., Helmstaedt, H.H. (Eds.), Western Superior Transect Fifth Annual Workshop. Lithoprobe Report 70, Lithoprobe Secretariat, University of British Columbia, pp. 66-75.

Frei, R., Rosing, M.T., 2001. The least radiogenic terrestrial leads: Implications for the early Archaean crustal evolution and hydrothermal-metasomatic processes in the Isua Supracrustal Belt (West Greenland). Chem. Geol. 181, 47-66.

Goolaerts, A., Mattielli, N., de Jong, J., Weis, D., Scoates, J.S., 2004. $\mathrm{Hf}$ and $\mathrm{Lu}$ isotopic reference values for the zircon standard 91500 by MC-ICP-MS. Chem. Geol. 206, 1-9.

Henry, P., Stevensen, R.K., Larbi, Y., Gariepy, C., 2000. Nd isotopic evidence for early to late Archean (3.4-2.7 Ga) crustal growth in the western Superior province (Ontario Canada). Tectonophysics 322, 135-151.

Henry, P., Stevenson, R.K., Gariepy, C., 1998. Late Archean mantle composition and crustal growth in the Western Superior Province of Canada: Neodymium and lead isotopic evidence from the Wawa. Quetico and Wabigoon subprovinces. Geochim. Cosmochim. Acta 62, 143-157.

Jacobsen, S.B., Dymek, R.F., 1988. Nd and Sr isotope systematics of clastic metasediments from Isua, West Greenland: identification of pre-3. 8 Ga differentiated crustal components. J. Geophys. Res. 93, 338-354.

Jaffey, A.H., Flynn, K.F., Glendenin, L.E., Bentley, W.C., Essling, A.M., 1971. Precision measurement of half-lives and specific activities of ${ }^{235} \mathrm{U}$ and ${ }^{238} \mathrm{U}$. Phys. Rev. C4, 1889-1906.

Kamber, B.S., Collerson, K.D., Moorbath, S., Whitehouse, M.J., 2003a. Inheritance of early Archean $\mathrm{Pb}$-isotope variability from long-lived Hadean protocrust. Contrib. Mineral. Petrol. 145, 25-46.

Kamber, B.S., Greig, A., Scheonberg, R., Collerson, K.D., 2003 b. A refined solution to the Earth's hidden niobium: implications for evolution of continental crust and mode of core formation. Precambrian Res. 126, 289-308.

King, E.M., Valley, J.W., Davis, D.W., Edwards, G.R., 1998. Oxygen isotope ratios of Archean plutonic zircons from granitegreenstone belts of the Superior province: indicator of magmatic source. Precambrian Res. 92, 365-387.

Langford, F.F., Morin, J.A., 1976. The development of the Superior province of northwestern Ontario by merging island arcs. Am. J. Sci. 276, 1023-1034. 
Maas, R., Kinney, P.D., Williams, I.S., Froude, D.O., Compston, W., 1992. The Earth's oldest known crust: A geochronological and geochemical study of 3900-4200 Ma old detrital zircons from Mt. Narryar and Jack Hills, Western Australia. Geochim. Cosmochim. Acta 56, 1281-1300.

Mattinson, J.M., 1987. U-Pb ages of zircons: a basic examination of error propagation. Chem. Geol. 66, 151-162.

Melnyk, M., Davis, D.W., Cruden, A.R., Stern, R.A. U-Pb ages of magmatism constraining regional deformation in the Winnipeg River subprovince and Lake of the Woods greenstone belt: evidence for Archean terrane accretion in the western Superior province. Can. J. Earth Sci., submitted.

Mojzsis, S.J., Harrison, T.M., Pidgeon, R.T., 2001. Oxygen-isotope evidence from ancient zircons for liquid water at the Earth's surface 4,300 Myr age. Nature 409, 178-181.

Nagler, Th.F., Kramers, J.D., 1998. Nd isotopic evolution of the upper mantle during the Precambrian: models, data and the uncertainty of both. Precambrian Res. 91, 233-252.

Nowell, G.M., Parrish, R.R., 2001. Simultaneous acquisition of isotope compositions and parent/daughter ratios by non-isotope dilution solution-mode Plasma Ionisation Multi-collector Mass Spectrometry (PIMMS). In: Holland, G.P., Tanner, S.D. (Eds.), 7th International Conference on Plasma Source Mass Spectrometry. Royal Society of Chemistry, pp. 298-310.

Nowell, G.M., Kempton, P.D., Noble, S.R., Fitton, J.G., Saunders, A.D., Mahoney, J.J., Taylor, R.N., 1998. High precision Hf isotopic measurements of MORB and OIB by thermal ionization mass spectrometry: insights into the depleted mantle. Chem. Geol. 149, 211-233.

Patchett, P.J., 1983. Importance of the Lu-Hf isotopic system in studies of planetary chronology and chemical evolution. Geochim. Cosmochim. Acta 47, 81-91.

Percival, J.A., Williams, H.R., 1989. The late Archean Quetico accretionary complex, Superior province, Canada. Geology 17, 23-25.

Percival, J.A., Stern, R.A., Skulski, T., Card, K.D., Mortensen, J.K., Begin, N.J., 1994. Minto Block. Superior Province; missing link in deciphering assembly of the craton at $2.7 \mathrm{Ga}$. Geology 22 , 839-842.

Samson, S.D., D’Lemos, R.S., Blichert-Toft, J., Vervoort, J., 2003. $\mathrm{U}-\mathrm{Pb}$ geochronology and Hf-Nd isotopic compositions of the oldest Neoproterozoic crust within the Cadominian orogen: new evidence for a unique juvenile terrane. Earth Planet. Sci. Lett. 208, 165-180.

Scherer, E., Munker, C., Mezger, K., 2001. Calibration of the Lutetium-Hafnium clock. Science 293, 683-687.

Scherer, E., Metzger, K., Münker, C., 2003. The ${ }^{176}$ Lu decay constant discrepancy: terrestrial samples vs. meteorites. 66th Annual Meeting of the Meteoritical Society.

Shirey, S.B., Hanson, G.N., 1984. Mantle-derived Archean monzodiorites and trachyandesites. Nature 310, 222-224.

Siguigna, A.P., Larabee, A.J., Waddington, J.C., 1982. The half life of $176 \mathrm{Lu}$ by a $\gamma-\gamma$ coincidence measurement. Can. J. Phys. 60 , 361-364.

Skulski, T., Sanborn-Barrie, M., Stern, R., 1999. Continental margin and oceanic terranes in the Savant Lake-Sturgeon Lake greenstone belt, Ontario. In: Harrap, R.M., Helmstaedt, H.H. (Eds.), Western Superior Transect Fifth Annual Workshop. Litho- probe Report 70, Lithoprobe Secretariat, University of British Columbia, pp. 165-166.

Smith, P.E., Tatsumoto, M., Farquhar, R.M., 1987. Zircon Lu-Hf systematics and the evolution of the Archean crust in the southern Superior Province, Canada. Contrib. Mineral. Petrol. 97, 93104.

Söderlund, U., Patchett, P.J., Vervoort, J.D., Isachsen, C.E., 2003. The decay constant of ${ }^{176} \mathrm{Lu}$ determined from $\mathrm{Lu}-\mathrm{Hf}$ and $\mathrm{U}-\mathrm{Pb}$ isotope systematics of terrestrial Precambrian high temperature mafic intrusions. 66th Annual Meeting of the Meteoritical Society.

Stern, R.A., Bleeker, W., 1998. Age of the world's oldest rocks refined using Canada's SHRIMP: The Acasta gneiss complex, Northwest Territories, Canada. Geosci. Cananda 25, 27-31.

Stern, R.A., Hanson, G.N., Shirey, S.B., 1990. Petrogenesis of mantle-derived, LILE-enriched Archean monzodiorites and trachyandesites (sanukitoids) in southwestern Superior Province, Reply. Can. J. Earth Sci. 27, 1136-1137.

Stevenson, R., Henry, P., Gariepy, C., 1999. Assimilation-fractional crystallization origin of Archean sanukitoid suites: western Superior province, Canada. Precambrian Res. 96, 83-99.

Stevensen, R.K., Patchett, P.J., 1990. Implications for the evolution of continental crust from $\mathrm{Hf}$ isotopes of Archean detrital zircons. Geochim. Cosmochim. Acta 54, 1683-1697.

Stott, G.M., Corfu, F., 1991. Uchi subprovince. In: Thurston, P.C., Williams, H.R., Sutcliffe, R.H., Stott, G.M. (Eds.), Geology of Ontario, Special Volume 4, Part 1, Ontario Geological Survey, pp. 144-236.

Stott, G.M., Davis, D.W., 1999. Contributions to the tectonostratigraphic analysis of the Onaman-Tashota greenstone belt, eastern Wabigoon subprovince. In: Harrap, R.M., Helmstaedt, H.H. (Eds.), Western Superior Transect Fifth Annual Workshop. Lithoprobe Report 70, 122-123. Lithoprobe Secretariat, University of British Columbia.

Thurston, P.C., Chivers, K.M., 1990. Secular variation in greenstone sequence development emphasising Superior Province, Canada. Precambrian Res. 46, 21-58.

Thurston, P.C., Osmani, I.A., Stone, D., 1991. Northwestern Superior province: review and terrane analysis. In: Thurston, P.C., Williams, H.R., Sutcliffe, R.H., Stott, G.M. (Eds.), Geology of Ontario, Special Volume 4, Part 1, Ontario Geological Survey, pp. 80-142.

Tomlinson, K.Y., Davis, D.W., Stone, D., Hart, T.R., 2003. U-Pb age and $\mathrm{Nd}$ isotopic evidence for crustal recycling and Archean terrane development in the south-central Wabigoon subprovince, Canada. Contrib. Mineral. Petrol. 144, 684-702.

Tomlinson, K.Y., Davis, D.W., Percival, J.A., Hughes, D.J., Thurston, P.C., 2002. Mafic to felsic magmatism and crustal recycling in the Obonga Lake greenstone belt, Western Superior Province: evidence from geochemistry, $\mathrm{Nd}$ isotopes and $\mathrm{U}-\mathrm{Pb}$ geochronology. Precambrian Res. 114, 295-325.

Tomlinson, K.Y., Skulski, T., Whalen, J., Stott, G.M., Stone, D., Percival, J., 2001. Nd isotopic mapping of terrane boundaries in the Wabigoon and Winnipeg River subprovinces. In: Harrap, R.M., Helmstaedt, H.H. (Eds.), Western Superior Transect Sixth Annual Workshop, Lithoprobe Report 80, Lithoprobe Secretariat, University of British Columbia, pp. 4-7. 
Unruh, D.M., Stille, P., Patchett, P.J., Tatsumoto, M., 1984. Lu-Hf and Sm-Nd evolution in lunar mare basalts. J. Geophys. Res. 89, B459-B477.

Vervoort, J.D., Blichert-Toft, J., 1999. Evolution of the depleted mantle: Hf isotope evidence from juvenile rocks through time. Geochim. Cosmochim. Acta 63, 533-556.

Vervoort, J.D., Patchett, P.J., 1996. Behavior of hafnium and neodymium isotopes in the crust: constraints from Precambrian crustally derived granites. Geochim. Cosmochim. Acta 60, 3717-3733.

White, D.J., Musacchio, G., Helmstaedt, H.H., Harrap, R.M., Thurston, P.C., van der Velden, A., Hall, K., 2003. Images of a lower crustal oceanic slab: Direct evidence of tectonic accretion in the Archean western Superior province. Geology 31, 997-1000.

Wiedenbeck, M., Allé, P., Corfu, F., Griffin, W.L., Meier, M., Oberli, F., Von Quadt, A., Roddick, J.C., Spiegel, W., 1995. Three natural zircon standards for U-Th- $\mathrm{Pb}, \mathrm{Lu}-\mathrm{Hf}$, trace element and REE analysis. Geostandards Newslett. 19, 1-23.

Wilde, S.A., Valley, J.W., Peck, W.H., Graham, C.M., 2001. Evidence from detrital zircons for the existence of continental crust and oceans on the Earth 4.4 Gyr ago. Nature 409, 175178. 